\title{
Static, stability and dynamic analyses of second strain gradient elastic Euler-Bernoulli beams
}

Received: 8 February 2020 / Revised: 30 September 2020 / Accepted: 26 November 2020 / Published online: 15 February 2021 (C) The Author(s), under exclusive licence to Springer-Verlag GmbH, AT part of Springer Nature 2021

\begin{abstract}
A simplified second strain gradient Euler-Bernoulli beam theory with two non-classical elastic coefficients in addition to the classical constants is presented. The governing equation and the associated classical and non-classical boundary conditions are derived with the aid of variational principles. The simplified second strain gradient theory is governed by an eighth-order differential equation with displacement, slope, curvature and triple derivative of displacement as degrees of freedom. This theory can be reduced to the first strain gradient and classical Euler-Bernoulli beam theories. Analytical solutions for static behaviour, free vibration and stability analyses are presented for different boundary conditions and length scale parameters. Using the numerical Laplace transform, a spectral element is developed for dynamic analysis of a cantilever beam subjected to a Gaussian pulse. Further, spectrum and dispersion relations are derived to study wave propagation characteristics. The gradient effects on the structural response are assessed and compared with the corresponding first strain gradient and classical beam theories. Observations show that the second strain gradient theory exhibiting stiffer behaviour in comparison to the first strain gradient and classical theories. The beam deflection decreases whereas frequencies and buckling load increase for increasing values of the gradient coefficient in comparison to the first strain gradient and classical theories. The forced response for a finite beam reveals a decrease in the amplitude and a shift to smaller time values with an increase in the value of length scale parameter. Additionally, the second strain gradient beam shows a dispersive behaviour, and for a given frequency the wavenumber decreases and the phase speed increases with an increase in the length scale parameter as compared to the first strain gradient beam theory.
\end{abstract}

\section{Introduction}

In recent years, more efforts have been made to understand the mechanical behaviour of micro- and nanoscale structural systems due to their outstanding features and wide range of applications, especially in the field of micro and nano-electromechanical systems [1,2]. For efficient and optimal design of these structural systems, a comprehensive understanding of the discrete structural behaviour at micro/nanoscale is very critical. Various approaches have been proposed in the literature to accurately predict the discrete behaviour of structures at nanoscale. For example, the experimental approach has been used by many researchers and found to be very tedious and expensive $[3,4]$. As an alternative, atomistic and semi-atomistic approaches such as lattice dynamics and molecular dynamic simulation are developed [5-7]. However, due to high computational cost, these methods cannot be generalised for practical applications. Considering the computational cost and accuracy

Md. Ishaquddin ( $\varangle) \cdot$ S. Gopalakrishnan

Department of Aerospace Engineering, Indian Institute of Science, Bengaluru 560012, India

e-mail: ishaquddinmd@iisc.ac.in

S. Gopalakrishnan

e-mail: krishnan@iisc.ac.in 
aspects, continuum-based approaches have been developed, that assure reasonable accuracy with less computational efforts as compared to discrete or atomistic approaches. In particular, the non-classical continuum theories with micro-structural properties have proved to be very efficient for predicting the micro/nanoscale structural behaviour.

The classical continuum theories which are based on the concept of homogeneity and locality of stress are effective for macroscale modelling of structural systems. Nevertheless, they lack efficiency to model the micro/nanoscale systems due to the absence of micro-structure length scale parameters. For instance, these theories are incapable of describing size-dependent mechanical behaviour of structures with micro-structure effects; they introduce singularity in problems with localised deformations; they are ineffective in treating strain softening, phase transformation and instability problems; unable to predict the dispersive behaviour in granular media, etc. To capture these size-dependent phenomena accurately, generalised continuum theories or nonclassical continuum theories with micro-structure effects are developed. These theories are enriched versions of classical continuum theories incorporating higher-order gradients of stress/strain tensors in conjunction with the intrinsic length scale parameters which account for scale effects. For example, Cosserat elasticity theory [8], micropolar theory [9], non-local elasticity theory [10-12], strain gradient theory [13, 14], Mindlin's strain gradient theory $[15,16]$ and couple stress theory $[17,18]$. The aforementioned theories are difficult to be used in practical applications as they contain many non-classical elastic constants in addition to the classical constants. Subsequently, many simplified and practically feasible versions which contain at least one non-classical elastic constant of dimension length in addition to the classical Lamé constants, have been developed to investigate the non-local and micro-structural effects on the mechanical behaviour of small-scale structures. The non-classical theories which have become popular in recent decades and have been extensively used in the literature due to their simplicity are the non-local elasticity theory by Eringen et al. [11, 12], Mindlin's simplified strain gradient theory [19-21], modified strain gradient theory by Lam et al. [22] and modified couple stress theory by Yang et al. [23]. These non-classical theories are governed by higher order differential equations and are more complicated compared to their classical counterparts. They introduce additional non-classical degrees of freedom and boundary conditions. Many analytical models are reported in the literature based on these non-classical theories to study the scale effects on the structural response [24-28].

In the non-classical strain gradient theories, the strain energy density depends on the elastic strain, its gradients and non-classical parameters that account for scale effects [13, 15, 20]. Mindlin et al. [15] proposed a first strain gradient theory (form-II) which has five and seven non-classical coefficients for static and dynamic applications, respectively, for an isotropic elastic material. Considering the complexity in determining the nonclassical parameters and their application to practical problems, a simplified version is developed by altering the generalised strain gradient theory $[15,16]$. This simplified version of first strain gradient theory has one and two non-classical elastic coefficients for static and dynamic analysis, respectively [29-37]. Employing this simplified theory, many analytical and numerical models for structural systems have been reported in the literature to study the gradient effects on the structural behaviour [38-44]. Later, Mindlin et al. [45] proposed a second strain gradient elastic theory formulated using the strain tensor and its first and second gradients. This theory is more involved and contains sixteen non-classical elastic coefficients in addition to two Lamé constants. It has been applied to various engineering problems to study the material and structural behaviour [46-56]. To develop a practically feasible model, Lazar et al. [57] and Castrenze et al. [58] formulated a simplified second strain gradient theory. This theory contains two non-classical elastic constants in addition to the classical constants and is used for dislocation and defect problems [59-62].

In this paper, an Euler-Bernoulli beam model based on simplified second strain gradient theory is developed, which has two non-classical elastic constants in addition to the classical constants. This is an extension of the earlier work on first strain gradient theory for an Euler-Bernoulli beam to study the static, stability and dynamic behaviour [38, 39, 42, 43]. The governing equation and the associated classical and non-classical boundary conditions are derived using the variational principles [63, 64]. Numerical examples on static, free-vibration and stability analyses of beams are presented to assess the gradient effects on the structural behaviour. Dynamic response of a cantilever beam subjected to a transverse Gaussian pulse is studied by formulating a spectral element based on numerical Laplace transform (NLT). Also, the spectrum and dispersion relations are derived to assess the influence of length scale parameters on the wave propagation characteristics. It is observed that the second strain gradient Euler-Bernoulli beam theory is governed by an eighth-order differential equation and has displacement, slope, curvature and triple derivative of displacement as degrees of freedom. Further, the second strain gradient theory exhibits stiffer behaviour in comparison to the first strain gradient and classical theories. The beam deflection decreases whereas frequencies and buckling load increase for increasing values of the gradient coefficient as compared to the first strain gradient theory. The forced response reveals a decrease 
in the amplitude and shift towards smaller time values with increasing length scale parameters. Furthermore, the second strain gradient beam shows a dispersive behaviour, and for a given frequency, the wavenumber decreases and phase speed increases with an increase in the gradient coefficient as compared to the first strain gradient beam theory.

\section{Second strain gradient Euler-Bernoulli beam theory}

In the present study, a simplified second strain gradient micro-elasticity theory by Lazar et al. [57] is considered, which contains two classical and two non-classical material constants. The two classical material constants correspond to Lamé constants and non-classical constants are of dimension length which are introduced to account for gradient effects. For a second strain gradient Euler-Bernoulli beam, the constitutive relations are expressed as

$$
\begin{aligned}
\tau_{x} & =E \varepsilon_{x}, \quad \varsigma_{x}=g_{1}^{2} E \varepsilon_{x}^{\prime}, \quad \bar{\zeta}_{x}=g_{2}^{4} E \varepsilon_{x}^{\prime \prime}, \\
\sigma_{x} & =\tau_{x}-\varsigma_{x}^{\prime}+\bar{\zeta}_{x}^{\prime}=E\left(\varepsilon_{x}-g_{1}^{2} \varepsilon_{x}^{\prime \prime}+g_{2}^{4} \varepsilon_{x}^{\prime \prime \prime}\right), \\
\varepsilon_{x} & =-z \frac{\partial^{2} w(x, t)}{\partial x^{2}}
\end{aligned}
$$

where $\sigma_{x}$ is the total stress, $\tau_{x}$ is the classical Cauchy stress, $\zeta_{x}, \bar{\zeta}_{x}$ are the higher order double and triple stresses, respectively, related to the gradient elasticity theory and $\varepsilon_{x}$ is the axial strain. Furthermore, $w$ is the transverse displacement of the beam, $z$ is the coordinate in the thickness direction, $E$ is the elastic Young's modulus, $g_{1}$, $g_{2}$ are the strain gradient constants of dimension length. The primes indicate order of derivative with respect to $x$. Based on the above constitutive relations, the strain energy for second strain gradient Euler-Bernoulli beam considering the effect of axial compressive force $P$ is written as

$$
U=\frac{1}{2} \int_{0}^{L} E I\left[\left(w^{\prime \prime}\right)^{2}+g_{1}^{2}\left(w^{\prime \prime \prime}\right)^{2}+g_{2}^{4}\left(w^{i v}\right)^{2}\right]-\frac{1}{2} \int_{0}^{L} P\left(w^{\prime}\right)^{2} \mathrm{~d} x,
$$

where $L$ is the length of the beam and $I$ is the second moment of area of cross section. The work done by the external applied load is given by

$$
W=-\int_{0}^{L} q(x) w \mathrm{~d} x-[V w]_{0}^{L}+\left[M w^{\prime}\right]_{0}^{L}+\left[\bar{M} w^{\prime \prime \prime}\right]_{0}^{L}+\left[\overline{\bar{M}} w^{\prime \prime \prime}\right]_{0}^{L}
$$

where $q$ is the transverse load, $V$ and $M$ are shear force and bending moment, $\bar{M}$ and $\overline{\bar{M}}$ are the double and triple moments acting on the beam. The kinetic energy is given as

$$
K=\frac{1}{2} \int_{0}^{L} m \dot{w}^{2} \mathrm{~d} x
$$

where $m=\rho A$ is the mass per unit length, $\rho$ is the density and the over dot indicates the derivative with respect to time $t$. Taking the first variation of the strain energy given in Eq. (2) and performing integration by parts, the following expression is obtained

$$
\begin{aligned}
\delta U= & \int_{0}^{L}\left[E I\left[w^{i v}-g_{1}^{2} w^{v i}+g_{2}^{4} w^{v i i i}\right)+P w^{\prime \prime}\right] \delta w \mathrm{~d} x \\
& -\left[\left\{E I\left[w^{\prime \prime \prime}-g_{1}^{2} w^{v}+g_{2}^{4} w^{v i i}\right)+P w^{\prime}\right\} \delta w\right]_{0}^{L}+\left[\left\{E I\left[w^{\prime \prime}-g_{1}^{2} w^{i v}+g_{2}^{4} w^{v i}\right)\right\} \delta w^{\prime}\right]_{0}^{L} \\
& +\left[\left\{E I\left[g_{1}^{2} w^{\prime \prime \prime}-g_{2}^{4} w^{v}\right)\right\} \delta w^{\prime \prime}\right]_{0}^{L}+\left[\left\{E I g_{2}^{4} w^{i v}\right\} \delta w^{\prime \prime \prime}\right]_{0}^{L} .
\end{aligned}
$$

Similarly, the variation of external work done is given by

$$
\delta W=-\int_{0}^{L} q(x) \delta w \mathrm{~d} x-[V \delta w]_{0}^{L}+\left[M \delta w^{\prime}\right]_{0}^{L}+\left[\bar{M} \delta w^{\prime \prime}\right]_{0}^{L}+\left[\overline{\bar{M}} \delta w^{\prime \prime \prime}\right]_{0}^{L} .
$$


Now taking the variation of kinetic energy with respect to time and performing integration by parts over the time interval $\left(t_{1}, t_{2}\right)$, we obtain

$$
\int_{t_{1}}^{t_{1}} \delta K \mathrm{~d} t=-\int_{t_{1}}^{t_{2}} \int_{0}^{L} m \ddot{w} \delta w \mathrm{~d} x \mathrm{~d} t+\int_{0}^{L} m[\dot{w} \delta w]_{t_{1}}^{t_{2}} \mathrm{~d} x .
$$

Substituting Eqs. (5)-(7) in Hamilton's principle [81]

$$
\delta \int_{t_{1}}^{t_{2}}[K-(U+W)] \mathrm{d} t=0
$$

we get

$$
\begin{aligned}
& \int_{t_{1}}^{t_{2}} \int_{0}^{L}\left[E I\left[w^{i v}-g_{1}^{2} w^{v i}+g_{2}^{4} w^{v i i i}\right)-q(x)+m \ddot{w}+P w^{\prime \prime}\right] \delta w \mathrm{~d} x \mathrm{~d} t \\
& -\int_{t_{1}}^{t_{2}}\left[\left\{V-E I\left[w^{\prime \prime \prime}-g_{1}^{2} w^{v}+g_{2}^{4} w^{v i i}\right)-P w^{\prime}\right\} \delta w\right]_{0}^{L} \mathrm{~d} t \\
& -\int_{t_{1}}^{t_{2}}\left[\left\{M-E I\left[w^{\prime \prime}-g_{1}^{2} w^{i v}+g_{2}^{4} w^{v i}\right)\right\} \delta w^{\prime}\right]_{0}^{L} \mathrm{~d} t \\
& -\int_{t_{1}}^{t_{2}}\left[\left\{\bar{M}-E I\left[g_{1}^{2} w^{\prime \prime \prime}-g_{2}^{4} w^{v}\right)\right\} \delta w^{\prime \prime}\right]_{0}^{L} \mathrm{~d} t-\int_{t_{1}}^{t_{2}}\left[\left\{\overline{\bar{M}}-\left[E I g_{2}^{4} w^{i v}\right)\right\} \delta w^{\prime \prime \prime}\right]_{0}^{L} \mathrm{~d} t \\
& -\int_{0}^{L} m[\dot{w} \delta w]_{t_{1}}^{t_{2}} \mathrm{~d} x=0 .
\end{aligned}
$$

It is to be noted that the integrand of the last term of Eq. (9) vanishes for the beam whose configurations at initial time $t_{1}$ and end time $t_{2}$ are prescribed. Hence

$$
\left[\dot{w}\left(x, t_{2}\right) \delta w\left(x, t_{2}\right)\right]-\left[\dot{w}\left(x, t_{1}\right) \delta w\left(x, t_{1}\right)\right]=0 .
$$

The variational statement in Eq. (9) implies that each term must be equal to zero, identically. Hence, the governing equation for a second strain gradient Euler-Bernoulli beam is obtained as:

$$
E I\left(w^{i v}-g_{1}^{2} w^{v i}+g_{2}^{4} w^{v i i i}\right)-q+P w^{\prime \prime}+m \ddot{w}=0
$$

and the associated boundary conditions are as: Classical:

$$
\begin{aligned}
& V=E I\left[w^{\prime \prime \prime}-g_{1}^{2} w^{v}+g_{2}^{4} w^{v i i}\right)-P w^{\prime}=0 \text { or } w=0, \quad \text { at } x=(0, L) \\
& M=E I\left[w^{\prime \prime}-g_{1}^{2} w^{i v}+g_{2}^{4} w^{v i}\right)=0 \text { or } w^{\prime}=0, \quad \text { at } x=(0, L)
\end{aligned}
$$

Non-classical:

$$
\begin{aligned}
& \bar{M}=E I\left[g_{1}^{2} w^{\prime \prime \prime}-g_{2}^{4} w^{v}\right)=0 \text { or } w^{\prime \prime}=0, \quad \text { at } x=(0, L) \\
& \overline{\bar{M}}=E I g_{2}^{4} w^{i v}=0 \text { or } w^{\prime \prime \prime}=0, \text { at } x=(0, L)
\end{aligned}
$$

when $g_{2}=0$, the second strain gradient theory reduces to first strain gradient theory and if $g_{1}=g_{2}=0$, the classical Euler-Bernoulli beam theory is obtained. The list of classical and non-classical boundary conditions employed in the present study for a second strain gradient Euler-Bernoulli beam is as follows:

Simply supported:

classical: $w=M=0, \quad$ non-classical: $w^{\prime \prime}=w^{\prime \prime \prime}=0$ at $x=(0, L)$

Clamped:

classical: $w=w^{\prime}=0$, non-classical: $w^{\prime \prime}=w^{\prime \prime \prime}=0$ at $x=(0, L)$

Cantilever:

classical: $w=w^{\prime}=0$ at $x=0 ; V=M=0$ at $x=L$

non-classical : $w^{\prime \prime}=w^{\prime \prime \prime}=0$ at $x=0 ; \bar{M}=\overline{\bar{M}}=0$ at $x=L$ 


\section{Analytical solutions for second strain gradient Euler-Bernoulli beam theory}

In this section, the analytical solutions for bending, free vibration and stability analyses of second strain gradient Euler-Bernoulli beam for different support conditions are obtained.

\subsection{Static analysis}

Consider a beam of length $L$ subjected to a uniformly distributed load (udl) $q$. To obtain the static deflections of the second gradient elastic Euler-Bernoulli beam which is governed by the equation

$$
E I\left[w^{i v}-g_{1}^{2} w^{v i}+g_{2}^{4} w^{v i i i}\right)-q=0,
$$

a solution of the form

$$
w(x)=a_{1}+a_{2} x+a_{3} x^{2}+a_{4} x^{3}+a_{5} e^{n_{1} x}+a_{6} e^{n_{2} x}+a_{7} e^{m_{1} x}+a_{8} e^{m_{2} x}-\frac{q x^{4}}{24 E I}
$$

is assumed, where

$$
\begin{aligned}
& n_{1}=\sqrt{\frac{g_{1}^{2}+\sqrt{g_{1}^{4}-4 g_{2}^{4}}}{2 g_{2}^{4}}}, \quad n_{2}=-\sqrt{\frac{g_{1}^{2}+\sqrt{g_{1}^{4}-4 g_{2}^{4}}}{2 g_{2}^{4}}}, \\
& m_{1}=\sqrt{\frac{g_{1}^{2}-\sqrt{g_{1}^{4}-4 g_{2}^{4}}}{2 g_{2}^{4}}}, \quad m_{2}=-\sqrt{\frac{g_{1}^{2}-\sqrt{g_{1}^{4}-4 g_{2}^{4}}}{2 g_{2}^{4}}},
\end{aligned}
$$

The constants $a_{1}-a_{8}$ are determined with the aid of the boundary conditions listed in Eqs. (12) and (13). After applying the boundary conditions the system of equations are expressed as:

$$
[K]\{\delta\}=\{f\},
$$

where $K$ is the stiffness matrix, $f$ is the force vector and $\{\delta\}=\left\{a_{1}, a_{2}, a_{3}, a_{4}, a_{5}, a_{6}, a_{7}, a_{8}\right\}^{T}$ is the unknown coefficient vector to be determined using the boundary conditions. The stiffness matrix $K$ is an extension of the earlier work on first strain gradient Euler-Bernoulli beam theory by Pegios et al. [42]. Once the unknown coefficients are determined, the displacement solution is obtained from Eq. (15). The slope, curvature and triple derivative of displacement at any point along the length of the beam can be computed by performing the first, second and third derivative of Eq. (15), respectively. The shear force, bending moment, double moment and triple moment are obtained by substituting Eq. (15) in Eqs. (12) and (13). The stiffness matrix and load vector for different boundary conditions are given in the Appendix.

\subsection{Free vibration analysis}

To obtain the natural frequencies of the second gradient elastic Euler-Bernoulli beam, a solution is assumed as

$$
w(x, t)=\bar{w}(x) e^{i \omega t} .
$$

Substituting the above solution in the following equation of motion:

$$
E I\left(w^{i v}-g_{1}^{2} w^{v i}+g_{2}^{4} w^{v i i i}\right)+m \ddot{w}=0,
$$

gives

$$
g_{2}^{4} \bar{w}^{v i i i}-g_{1}^{2} \bar{w}^{v i}+\bar{w}^{i v}-\frac{\omega^{2}}{\beta^{2}} \bar{w}=0,
$$


where $\beta^{2}=E I / m$. Equation (19) has the solution of the form

$$
\bar{w}(x)=\sum_{j=1}^{8} c_{i} e^{\alpha_{i} x},
$$

where $c_{i}$ are the unknown constants which are determined using boundary conditions and $\alpha_{i}$ are the roots of the characteristic equation

$$
g_{2}^{4} \alpha^{8}-g_{1}^{2} \alpha^{6}+\alpha^{4}-\frac{\omega^{2}}{\beta^{2}}=0 .
$$

After applying the boundary conditions given in Eqs. (12) and (13), we get

$$
[F(\omega)]\{c\}=\{0\} .
$$

For a non-trivial solution, the following condition is to be satisfied:

$$
\operatorname{det}[F(\omega)]=0 .
$$

The natural frequencies for second strain gradient Euler-Bernoulli beam for different support conditions are obtained by solving Eq. (23) using the procedure given in Kitahara et al. [80]. The respective frequency equations $F(\omega)$ are presented in the Appendix. The numerical procedure involves finding the minimum values of $D(\omega)=\ln |\operatorname{det}[F(\omega)]|$ for a series of frequencies $\omega$. The frequencies which make $D(\omega)$ minimum are the natural frequencies as illustrated in Fig. 4.

\subsection{Stability analysis}

To obtain the buckling load for a second strain gradient Euler-Bernoulli beam governed by

$$
E I\left(w^{i v}-g_{1}^{2} w^{v i}+g_{2}^{4} w^{v i i i}\right)+P w^{\prime \prime}=0,
$$

a solution is assumed as

$$
w(x)=b_{1}+b_{2} x+b_{3} e^{\bar{m}_{1} x}+b_{4} e^{\bar{m}_{2} x}+b_{5} e^{\bar{m}_{3} x}+b_{6} e^{\bar{n}_{1} x}+b_{7} e^{\bar{n}_{2} x}+b_{8} e^{\bar{n}_{3} x},
$$

where $b_{i}$ are the unknown constants which are determined through boundary conditions and $\bar{m}_{1,2,3}$ and $\bar{n}_{1,2,3}$ are six roots of the following characteristic equation:

$$
g_{2}^{4} y^{6}-g_{1}^{2} y^{4}+y^{2}+\frac{P}{E I}=0 .
$$

After applying the boundary conditions listed in Eqs. (12) and (13), the following expression is obtained:

$$
[G(P)]\{b\}=\{0\} .
$$

For non-trivial solution, the following condition is to be satisfied:

$$
\operatorname{det}[G(P)]=0,
$$

where $G(P)$ is the geometric stiffness matrix. Earlier, the geometric stiffness matrix for first strain gradient Euler-Bernoulli beam theory is developed by Pegios et al. [42]. The buckling load for second strain gradient Euler-Bernoulli beam with different boundary conditions is obtained by following the approach given by Kitahara et al. [80] and the associated geometric stiffness matrices $G(P)$ are presented in the Appendix. 


\subsection{Dynamic analysis}

In this section, a spectral element for dynamic analysis of second strain gradient Euler-Bernoulli beam using NLT is developed. The spectral element formulation is based on the Laplace transformation of the governing differential equation and boundary conditions. NLT is an efficient tool for dynamic analysis and used extensively in many applications [65-74]. The Laplace transform can be considered as Fourier transform of an exponential signal analysed in terms of sinusoids and exponentials. The Laplace transform is expressed as $[75,76]$

$$
\begin{aligned}
& \bar{f}(\eta+i \omega)=\int_{0}^{\infty}\left[f(t) e^{-\eta t}\right] e^{-i \omega t} \mathrm{~d} t, \\
& f(t)=\frac{e^{\eta t}}{2 \pi} \int_{-\infty}^{\infty}[\bar{f}(\eta+i \omega)] e^{i \omega t} \mathrm{~d} \omega,
\end{aligned}
$$

where $i=\sqrt{-1}, \omega$ is the angular frequency, $\eta$ is the Laplace variable, $N$ is the number of FFT sampling points and it is taken as an integer power of 2 for efficient computations, and $T$ is the time of observation or time window. It is to be noted that when $\eta=0$ Eqs. (29) and (30) reduce to the continuous Fourier transform and can be implemented in the discrete framework using Discrete Fourier Transform (DFT). The advantage of the real part of the Laplace variable $\eta$ is that it acts like a damping parameter and damps out all the responses beyond the chosen time window $T$, thereby eliminating the signal wraparound problem which normally arises when FFT is used for transforming the variables [77]. The discrete form of the forward and inverse Laplace transforms of Eqs. (29) and (30) can be expressed as:

$$
\begin{gathered}
\bar{f}(\eta+i k \Delta f)=\Delta t \sum_{n=0}^{N-1}\left[f(n \Delta t) e^{(-\eta)(n \Delta t)}\right] e^{-i(2 \pi k \Delta f)(n \Delta t),} \\
\left.f(n \Delta t)=e^{\eta(n \Delta t)}\left[\Delta f \sum_{k=0}^{N-1} \bar{f}(\eta+i k \Delta f)\right] e^{i(2 \pi k \Delta f)(n \Delta t)}\right] .
\end{gathered}
$$

The term inside the square bracket in Eq. (32) allows to use the FFT algorithm [78, 79]. The FFT-based implementation requires that the real part of the Laplace transform variable $\eta$ be a function of the time window $T$ and the number of FFT points $N$. Two different empirical formulae are suggested in the literature for the real part of the Laplace variable and given as $\eta_{\text {Wilcox }}=2 \Delta \omega=2 \pi / T$ [73] and $\eta_{\text {Wedepohl }}=2 \ln (N) / T$ [74], where $\Delta \omega$ is the spectrum integration step. In this research the value of the Laplace variable is taken as $\eta_{\text {Wedepohl }}=2 \ln (N) / T$. Assuming a spectral solution of the form

$$
w(x, t)=\tilde{w}(x, s) e^{-(i k x-s t)}
$$

and substituting in the equation of motion (18), we get a characteristic equation in the Laplace domain as

$$
g_{2}^{4} k^{8}-g_{1}^{2} k^{6}+k^{4}-\frac{s^{2}}{\beta^{2}}=0 .
$$

The above equation gives 4 pairs of roots $\pm \sqrt{k_{1}}, \pm \sqrt{k_{2}}, \pm \sqrt{k_{3}}$ and $\pm \sqrt{k_{4}}$, where $k$ is the wavenumber, $s$ is Laplace variable defined as $s=(\eta+i \omega)$.

Now, the generic displacement vector can be rewritten using Eq. (33) and the above roots as:

$$
\tilde{w}(x, s)=\sum_{j=1}^{8} d_{j} e^{-i k_{j} x} .
$$

The second strain gradient Euler-Bernoulli beam has $w, w^{\prime}, w^{\prime \prime}$ and $w^{\prime \prime \prime}$ as degrees of freedom per node and for a 2-noded element it has eight degrees of freedom per element. The displacement field in Eq. (35) in terms of degrees of freedom can be expressed as

$$
\Delta=\left\{\begin{array}{c}
w \\
w^{\prime} \\
w^{\prime \prime} \\
w^{\prime \prime \prime}
\end{array}\right\}=\left[\begin{array}{ccc}
A_{11} & \ldots & A_{18} \\
A_{21} & \ldots & A_{28} \\
A_{31} & \ldots & A_{38} \\
A_{41} & \ldots & A_{48}
\end{array}\right][\Gamma]\left\{\begin{array}{c}
d_{1} \\
\cdot \\
\cdot \\
\cdot \\
d_{8}
\end{array}\right\}=\left[\bar{K}_{1}\right]\{\delta\},
$$


where $\delta=\left\{d_{1} \ldots d_{8}\right\}^{T}$ are unknown wave coefficients, $A_{4 \times 8}$ is the amplitude matrix and $\Gamma_{8 \times 8}$ is a diagonal matrix defined as

$$
\begin{gathered}
A_{1 j}=1 ; \quad A_{2 j}=-i k_{j} ; \quad A_{3 j}=-k_{j}^{2} ; \quad A_{4 j}=i k_{j}^{3}, \\
\Gamma_{j j}=e^{-i k_{j} x} \quad(j=1,8) .
\end{gathered}
$$

Evaluating $\Delta$ at the two boundary nodes of the element $x=(0, L)$, we obtain

$$
\Delta^{e}=\left\{\begin{array}{l}
\bar{K}_{1(x=0)} \\
\bar{K}_{1(x=L)}
\end{array}\right\}\{\delta\}=\left[\bar{K}_{2}\right]\{\delta\},
$$

where $\Delta^{e}=\left\{w_{1}, w_{1}^{\prime}, w_{1}^{\prime \prime}, w_{1}^{\prime \prime \prime}, w_{2}, w_{2}^{\prime}, w_{2}^{\prime \prime}, w_{2}^{\prime \prime \prime}\right\}^{T}$ contains all degrees of freedom of the element, $\bar{K}_{2}$ is a $8 \times 8$ non-singular complex matrix and the superscript $e$ indicates the element. Eliminating the unknown coefficient vector $\delta$ using Eqs. (36) and (38), we get the displacement field in terms of element nodal degrees of freedom as

$$
\Delta=\left[\bar{K}_{1}\right]\left[\bar{K}_{2}\right]^{-1} \Delta^{e}=\left[S_{f}\right] \Delta^{e},
$$

where $S_{f}$ is the element shape function matrix. To obtain the dynamic stiffness matrix, the nodal forces $\hat{f}=\{V, M, \bar{M}, \overline{\bar{M}}\}^{T}$ given in Eqs. (12) and (13) are expressed in the Laplace domain by using Eq. (35) and we get

$$
\hat{f}=\left\{\begin{array}{c}
V \\
M \\
\bar{M} \\
\overline{\bar{M}}
\end{array}\right\}=\left[\begin{array}{lll}
R_{11} & \ldots & R_{18} \\
R_{21} & \ldots & R_{28} \\
R_{31} & \ldots & R_{38} \\
R_{41} & \ldots & R_{48}
\end{array}\right][\Gamma]\left\{\begin{array}{c}
d_{1} \\
\cdot \\
\cdot \\
\dot{d_{8}}
\end{array}\right\}=\left[\bar{K}_{f}\right]\{\delta\},
$$

where $R$ is given by

$$
\begin{aligned}
& R_{1 j}=-E I\left\{i k_{j}^{3}+g_{1}^{2} i k_{j}^{5}+g_{2}^{4} i k_{j}^{7}\right\} ; \quad R_{2 j}=-E I\left\{k_{j}^{2}+g_{1}^{2} k_{j}^{4}+g_{2}^{4} k_{j}^{6}\right\} ; \\
& R_{3 j}=E I\left\{g_{1}^{2} i k_{j}^{3}+g_{2}^{4} i k_{j}^{4}\right\} ; \quad R_{4 j}=E I\left\{g_{2}^{4} k_{j}^{4}\right\} \quad(j=1,8) .
\end{aligned}
$$

Evaluating the nodal forces at the element boundaries $x=(0, L)$, we get

$$
\hat{f}^{e}=\left\{\begin{array}{l}
\bar{K}_{f(x=0)} \\
\bar{K}_{f(x=L)}
\end{array}\right\}\{\delta\}=\left[\bar{K}_{3}\right]\{\delta\} .
$$

Combining Eqs. (38) and (42), the relationship between the element nodal forces and displacements is obtained as

$$
\hat{f}^{e}=\left[\bar{K}_{3}\right]\left[\bar{K}_{2}\right]^{-1} \Delta^{e}=\left[K_{d s}\right] \Delta^{e},
$$

where $\left[K_{d s}\right]_{8 \times 8}$ is the dynamic stiffness matrix in the Laplace domain computed at each frequency. Using Eqs. (45) and (44), the dynamic response $w(x, t)$ from the Laplace domain to the time domain is obtained as:

$$
w(x, t)=e^{\eta(n \Delta t)}\left[\Delta \hat{f}^{e} \sum_{n=0}^{N-1}\left[K_{d s}\left(\eta+i k \Delta \hat{f}^{e}\right)\right]^{-1} \hat{f}^{e}\left(\eta+i k \Delta \hat{f}^{e}\right) e^{i\left(2 \pi k \Delta \hat{f}^{e}\right)\left(n \Delta \hat{f}^{e}\right)}\right],
$$

where $\hat{f}^{e}\left(\eta+i k \Delta \hat{f}^{e}\right)$ is obtained using the forward FFT as:

$$
\hat{f}^{e}\left(\eta+i k \Delta \hat{f}^{e}\right)=\Delta t \sum_{n=0}^{N-1}\left[\hat{f}^{e}(n \Delta t) e^{-\eta n \Delta t}\right] e^{-i\left(2 \pi k \Delta \hat{f}^{e}\right)(n \Delta t)} .
$$




\subsection{Wave propagation analysis}

For harmonic wave propagation in a second strain gradient Euler-Bernoulli beam, a solution of the following form is assumed:

$$
w(x, t)=w_{o} e^{-i(k x-\omega t)},
$$

where $w_{o}, k$ and $\omega$ are amplitude, wave number and angular frequency of the wave motion, respectively. The imaginary number is defined as $i=\sqrt{-1}$. Substituting the above solution into the homogeneous differential equation given by Eq. (18), the following characteristic equation is obtained

$$
g_{2}^{4} E I k^{8}+g_{1}^{2} E I k^{6}+E I k^{4}-\rho A \omega^{2}=0 .
$$

The spectrum relation can be obtained from the above equation as [71]

$$
\omega=\sqrt{\frac{g_{2}^{4} E I k^{8}+g_{1}^{2} E I k^{6}+E I k^{4}}{\rho A}} .
$$

the phase speed is given by [71]

$$
\frac{\omega}{k}=\sqrt{\frac{g_{2}^{4} E I k^{6}+g_{1}^{2} E I k^{4}+E I k^{2}}{\rho A}},
$$

and the group speed is [71]

$$
\frac{d \omega}{d k}=\frac{4 g_{2}^{4} E I k^{5}+3 g_{1}^{2} E I k^{3}+2 E I k}{\sqrt{\rho A\left(g_{2}^{4} E I k^{4}+g_{1}^{2} E I k^{2}+E I\right)}} .
$$

\section{Numerical results and discussion}

In this section, numerical examples on bending, free vibration, stability, transient and wave propagation analysis are presented to assess the influence of length scale parameters on the response of a second strain gradient Euler-Bernoulli beam. The results are compared with those of classical and first strain gradient theories for different boundary conditions and length scale parameters $g_{1} / L$ and $g_{2} / L$. For ease of comparison, we designate the first strain gradient model as $\mathrm{Sg}$-I and the second strain gradient model as $\mathrm{Sg}$-II. The numerical data used for the analysis of beams is as follows: Length $L=1 \mu \mathrm{m}$, depth $d=0.1 \mu \mathrm{m}$, width $b=0.1 \mu \mathrm{m}$, Young's modulus $E=30 \times 10^{6} \mathrm{GPa}$, Poisson's ratio $v=0.3$, density $\rho=2700 \mathrm{~kg} / \mathrm{m}^{3}$ and load $q=1 \mu \mathrm{N}$.

\subsection{Static analysis of gradient elastic beams}

The static analysis of gradient elastic beams subjected to udl is presented herein. Three support conditions are considered, simply supported, clamped and cantilever. The non-dimensional deflection $\bar{w}=100 \mathrm{EIw} / q L^{4}$ is compared for three different combinations of length scale values, $g_{1} / L=0.1, g_{2} / L=0.1, g_{1} / L=$ $0.15, g_{2} / L=0.1$ and $g_{1} / L=0.1, g_{2} / L=0.15$. In Fig.1, the non-dimensional deflection obtained using the Sg-II model is compared with the Sg-I $\left(g_{1} / L=0.1, g_{2} / L=0\right)$ and the classical $\left(g_{1} / L=g_{2} / L=0\right)$ model for a simply supported beam. It is observed that the $\mathrm{Sg}$-II model shows lesser deflection than that of the $\mathrm{Sg}$-I and the classical model. For a given value of $g_{1} / L=0.1$, the $\mathrm{Sg}$-II model deflection decreases with increasing $g_{2} / L$. Similarly, for a given value of $g_{2} / L=0.1$, the Sg-II model deflection decreases with increasing $g_{1} / L$. However, the influence of $g_{2} / L$ is more significant on the deflection as compared to $g_{1} / L$. In Figs. 2 and 3, a similar stiffer behaviour is exhibited by the $\mathrm{Sg}$-II model for clamped and cantilever beams as compared to the Sg-I and classical model. Based on the above observations, it can be stated that the Sg-II model shows a stiffer behaviour in comparison to the classical and the Sg-I model. Further, the classical and the Sg-II model form the upper and lower bounds, respectively, for the Sg-I model for a given value of $g_{1} / L$. The parameter $g_{2}$ introduced into the second strain gradient theory has the effect of stiffening the behaviour. 


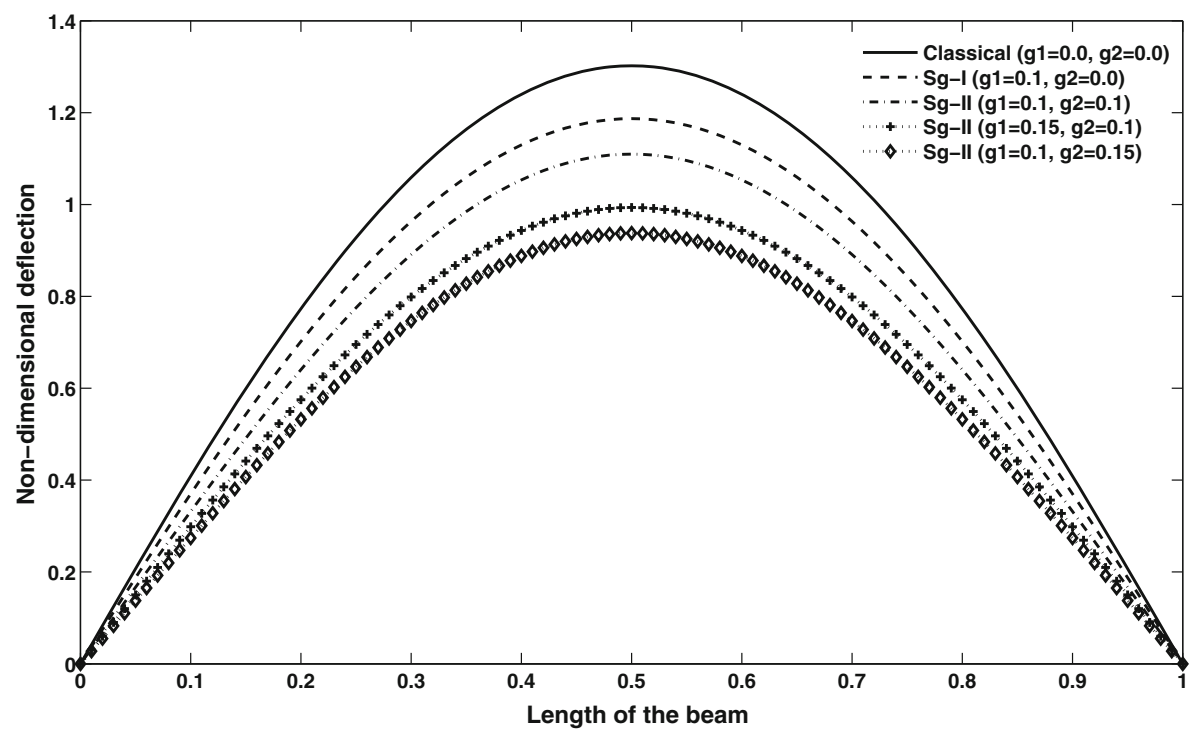

Fig. 1 Non-dimensional deflection variation for a simply supported beam under a udl

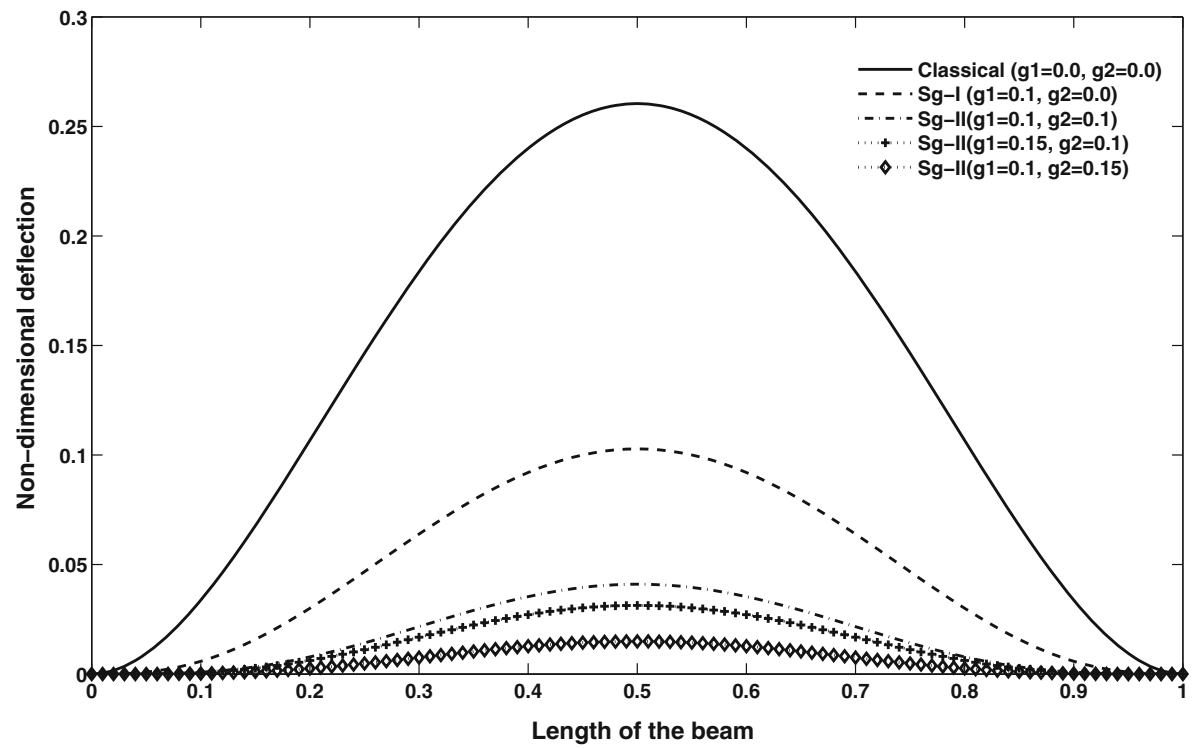

Fig. 2 Non-dimensional deflection variation for a clamped beam under a udl

\subsection{Free vibration analysis of gradient elastic beams}

The effect of length scale parameters on the natural frequencies obtained for a second strain gradient Euler-Bernoulli beam is studied. Three different boundary conditions are considered in this analysis: simply supported, clamped and cantilever. The frequencies are non-dimensional as: $\bar{\omega}=\omega L^{2} \sqrt{\rho A / E I}$ and are compared for two different combinations of length scale values, $g_{1} / L=0.1, g_{2} / L=0.1$ and $g_{1} / L=0.1, g_{2} / L=0.15$. In Fig. 4 , a plot of $\log |F(\omega)|$ versus $\omega$ is shown for a simply supported beam and the first three frequencies are identified, which are obtained using the procedure described in Kitahara et al. [80]. In Table 1, first six frequencies for a simply supported beam obtained using the classical, $\mathrm{Sg}$-I and $\mathrm{Sg}$-II beam models are tabulated. It can be observed that the $\mathrm{Sg}$-II model shows higher frequencies than the classical $\left(g_{1} / L=g_{2} / L=0\right)$ and the $\mathrm{Sg}-\mathrm{I}\left(g_{1} / L=0.1, g_{2} / L=0\right)$ models. For a given value of $g_{1} / L=0.1$, the frequencies obtained using the $\mathrm{Sg}$-II model increases with an increase in $g_{2} / L$. As behaviour in frequencies 


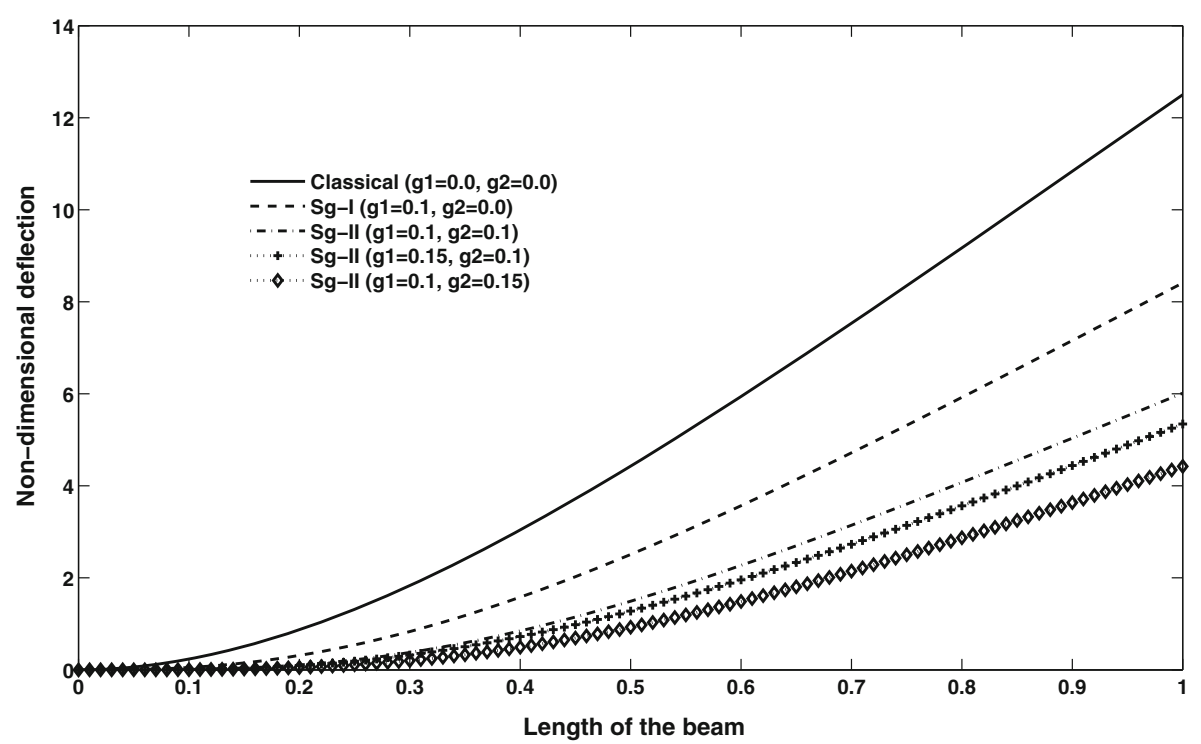

Fig. 3 Non-dimensional deflection variation for a cantilever beam under a udl

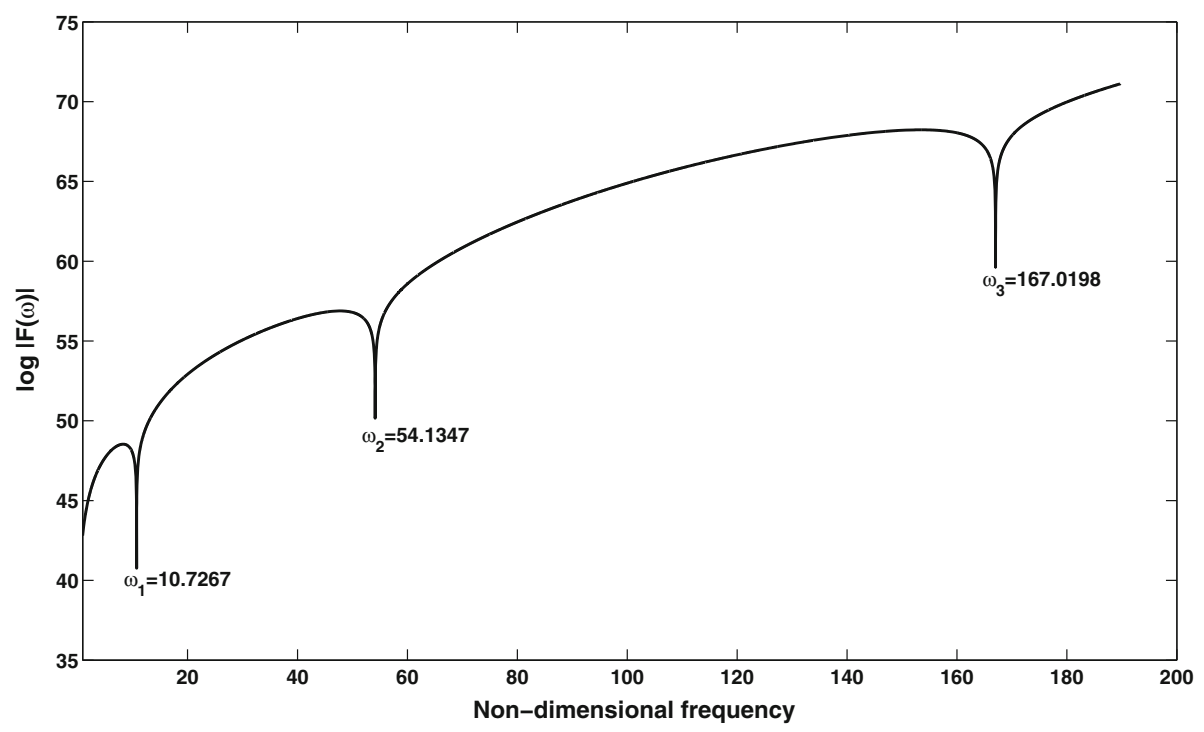

Fig. 4 First three non-dimensional frequencies for a second strain gradient simply supported beam $\left(g_{1} / L=g_{2} / L=0.1\right)$

is seen in Tables 2 and 3, for clamped and cantilever beams, respectively. Hence, the frequencies obtained using the $\mathrm{Sg}$-II model are higher than the Sg-I model for a given value of $g_{1} / L$.

\subsection{Stability analysis of gradient elastic beams}

In Table 4, the non-dimensional buckling load $\bar{P}_{c r}=P_{c r} L^{2} / E I$ obtained for simply supported, clamped and cantilever beams is presented. It can be observed that the Sg-II model shows higher buckling load than the classical $\left(g_{1} / L=g_{2} / L=0\right)$ and the Sg-I $\left(g_{1} / L=0.1, g_{2} / L=0\right)$ models for all the boundary conditions. The buckling load obtained using the Sg-II model increases with an increase in $g_{2} / L$ for a fixed $g_{1} / L$ value. 
Table 1 Comparison of first six non-dimensional natural frequencies for a simply supported beam

\begin{tabular}{lllll}
\hline $\begin{array}{l}\text { Freq. } \\
\bar{\omega}\end{array}$ & $\begin{array}{l}\text { Classical } \\
\left(\frac{g_{1}}{L}=\frac{g_{2}}{L}=0\right)\end{array}$ & $\begin{array}{l}\text { Sg-I } \\
\left(\frac{g_{1}}{L}=0.1, \frac{g_{2}}{L}=0\right)\end{array}$ & $\begin{array}{l}\text { Sg-II } \\
\left(\frac{g_{1}}{L}=\frac{g_{2}}{L}=0.1\right)\end{array}$ & $\begin{array}{l}\text { Sg-II } \\
\left(\frac{g_{1}}{L}=0.1, \frac{g_{2}}{L}=0.15\right)\end{array}$ \\
\hline $\bar{\omega}_{1}$ & 9.8696 & 10.3452 & 10.7267 & 11.6947 \\
$\bar{\omega}_{2}$ & 39.4784 & 46.6243 & 54.1347 & 73.9808 \\
$\bar{\omega}_{3}$ & 88.8264 & 122.0601 & 167.0198 & 275.7272 \\
$\bar{\omega}_{4}$ & 157.9137 & 253.6045 & 412.9814 & 762.4188 \\
$\bar{\omega}_{5}$ & 246.7401 & 459.4536 & 880.3240 & 1732.1901 \\
$\bar{\omega}_{6}$ & 355.3057 & 758.1478 & 1680.7079 & 3435.2844 \\
\hline
\end{tabular}

Table 2 Comparison of first six non-dimensional natural frequencies for a clamped beam

\begin{tabular}{lllll}
\hline $\begin{array}{l}\text { Freq. } \\
\bar{\omega}\end{array}$ & $\begin{array}{l}\text { Classical } \\
\left(\frac{g_{1}}{L}=\frac{g_{2}}{L}=0\right)\end{array}$ & $\begin{array}{l}\text { Sg-I } \\
\left(\frac{g_{1}}{L}=0.1, \frac{g_{2}}{L}=0\right)\end{array}$ & $\begin{array}{l}\text { Sg-II } \\
\left(\frac{g_{1}}{L}=\frac{g_{2}}{L}=0.1\right)\end{array}$ & $\begin{array}{l}\text { Sg-II } \\
\left(\frac{g_{1}}{L}=0.1, \frac{g_{2}}{L}=0.15\right)\end{array}$ \\
\hline $\bar{\omega}_{1}$ & 22.0266 & 35.8934 & 57.2239 & 95.1074 \\
$\bar{\omega}_{2}$ & 61.6850 & 108.9316 & 194.1768 & 353.5243 \\
$\bar{\omega}_{3}$ & 120.9026 & 239.1782 & 484.3670 & 940.2738 \\
$\bar{\omega}_{4}$ & 199.88595 & 444.2282 & 1021.5128 & 2067.2318 \\
$\bar{\omega}_{5}$ & 298.5555 & 742.4006 & 1923.0803 & 3998.0907 \\
$\bar{\omega}_{6}$ & 416.9908 & 1152.2065 & 3329.6826 & 7048.6249 \\
\hline
\end{tabular}

Table 3 Comparison of first six non-dimensional natural frequencies for a cantilever beam

\begin{tabular}{lllll}
\hline $\begin{array}{l}\text { Freq. } \\
\bar{\omega}\end{array}$ & $\begin{array}{l}\text { Classical } \\
\left(\frac{g 1}{L}=\frac{g_{2}}{L}=0\right)\end{array}$ & $\begin{array}{l}\text { Sg-I } \\
\left(\frac{g_{1}}{L}=0.1, \frac{g_{2}}{L}=0\right)\end{array}$ & $\begin{array}{l}\text { Sg-II } \\
\left(\frac{g_{1}}{L}=\frac{g_{2}}{L}=0.1\right)\end{array}$ & $\begin{array}{l}\text { Sg-II } \\
\left(\frac{g_{1}}{L}=0.1, \frac{g_{2}}{L}=0.15\right)\end{array}$ \\
\hline $\bar{\omega}_{1}$ & 3.5156 & 4.0373 & 5.1059 & 5.9669 \\
$\bar{\omega}_{2}$ & 22.0336 & 28.4554 & 34.8492 & 43.4446 \\
$\bar{\omega}_{3}$ & 61.7010 & 38.4899 & 115.6445 & 158.8339 \\
$\bar{\omega}_{4}$ & 120.9026 & 87.8028 & 285.8348 & 437.4657 \\
$\bar{\omega}_{5}$ & 199.8595 & 194.5272 & 610.9849 & 1037.4652 \\
$\bar{\omega}_{6}$ & 298.5555 & 365.5267 & 1182.5574 & 2175.0149 \\
\hline
\end{tabular}

Table 4 Comparison of non-dimensional buckling load for different support conditions

\begin{tabular}{lllll}
\hline $\begin{array}{l}\text { Support } \\
\text { condition }\end{array}$ & $\begin{array}{l}\text { Classical } \\
\left(\frac{g_{1}}{L}=\frac{g_{2}}{L}=0\right)\end{array}$ & $\begin{array}{l}\text { Sg-I } \\
\left(\frac{g_{1}}{L}=0.1, \frac{g_{2}}{L}=0\right)\end{array}$ & $\begin{array}{l}\text { Sg-II } \\
\left(\frac{g_{1}}{L}=\frac{g_{2}}{L}=0.1\right)\end{array}$ & $\begin{array}{l}\text { Sg-II } \\
\left(\frac{g_{1}}{L}=0.1, \frac{g_{2}}{L}=0.15\right)\end{array}$ \\
\hline Cantilever & 2.4674 & 3.1013 & 3.7043 & 4.4148 \\
Pinned & 9.8696 & 10.8436 & 11.6498 & 13.8331 \\
Clamped & 39.4784 & 83.2918 & 172.7872 & 460.3418 \\
\hline
\end{tabular}

\subsection{Dynamic analysis of gradient elastic beam}

The gradient effects on the dynamic response of the Sg-II model is studied for a cantilever beam subjected to a transverse tip Gaussian force as shown in Fig. 5. The response is obtained at the tip for two different combinations of length scale values, $g_{1} / L=0.1, g_{2} / L=0.1$ and $g_{1} / L=0.1, g_{2} / L=0.15$. In Fig. 6, the velocity response is plotted for classical, $\mathrm{Sg}-\mathrm{I}\left(g_{1} / L=0.1, g_{2} / L=0\right)$ and $\mathrm{Sg}$-II models. It is observed that the response obtained for $\mathrm{Sg}$-II model shows higher dispersion behaviour as compared to the classical and $\mathrm{Sg}$-I models and it increases with increasing the $g_{2} / L$ value for a given $g_{1} / L$. Furthermore, the Sg-II response shifts towards the lower time scale values and also the amplitude decreases with increasing length scale parameter as compared to the classical and the Sg-I model.

\subsection{Wave propagation analysis of gradient elastic beams}

The wave propagation characteristics of the Sg-II model is studied for three sets of length scale parameters $g_{1} / L=0.05, g_{2} / L=0.05 ; g_{1} / L=0.05, g_{2} / L=0.075$ and $g_{1} / L=0.05, g_{2} / L=0.1$. In Fig. 7 the 


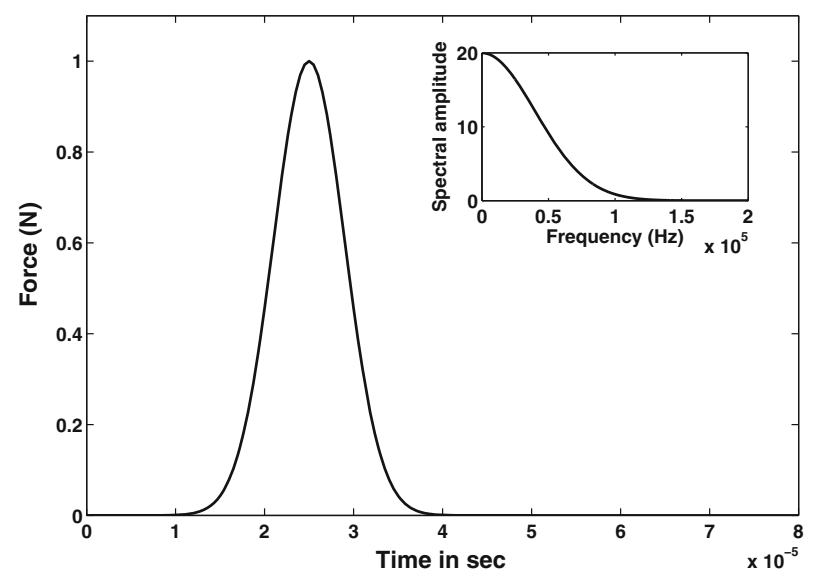

Fig. 5 The Gaussian pulse and its frequency content

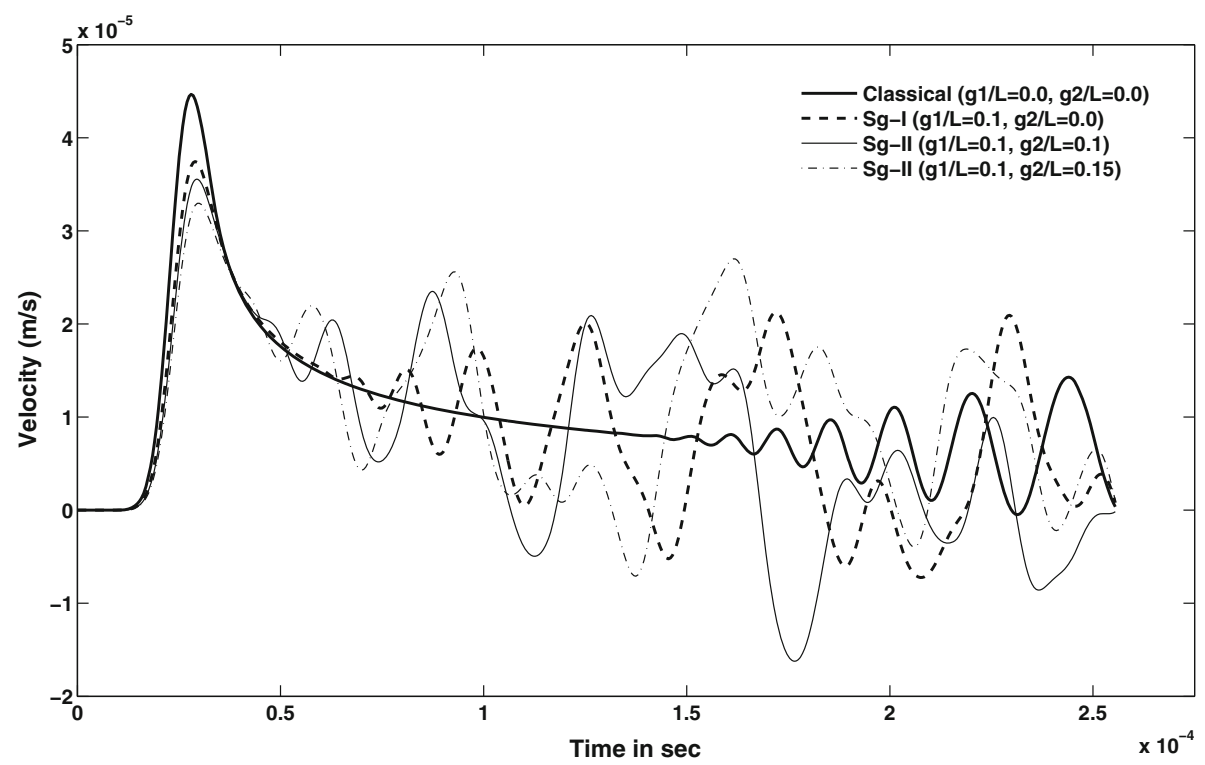

Fig. 6 Comparison of transverse velocity response at the tip of a cantilever beam subjected to a Gaussian pulse

spectrum relation for classical, $\mathrm{Sg}$-I $\left(g_{1} / L=0.05, g_{2} / L=0\right)$ and $\mathrm{Sg}$-II models is plotted. The $\mathrm{Sg}$-II model shows a lower wave number as compared to classical and the Sg-I models for $\omega \geq 0.5 \mathrm{kHz}$. As $g_{2} / L$ increases, the wave number decreases for the $\mathrm{Sg}$-II model for a chosen $g_{1} / L$ value. In Fig. 8 the dispersion relation is plotted and compared for all the three beam models. The Sg-II model exhibits a dispersive behaviour similar to the Sg-I model. The non-dimensional phase speed is higher for the Sg-II model in comparison to the classical and $\mathrm{Sg}$-I models for a given $g_{1} / L$ and $\omega \geq 0.5 \mathrm{kHz}$.

\section{Conclusion}

An Euler-Bernoulli beam model is developed based on the simplified second strain gradient theory which contains two non-classical elastic constants in-addition to the classical constants. The governing equation and associated classical and non-classical boundary conditions are derived with the aid of variational principles. The simplified second strain gradient theory can be reduced to first strain gradient and classical theories and is governed by eighth-order differential equation with displacement, slope, curvature and triple derivative of displacement as degrees of freedom. Analytical solutions for static, free vibration and stability analysis are 


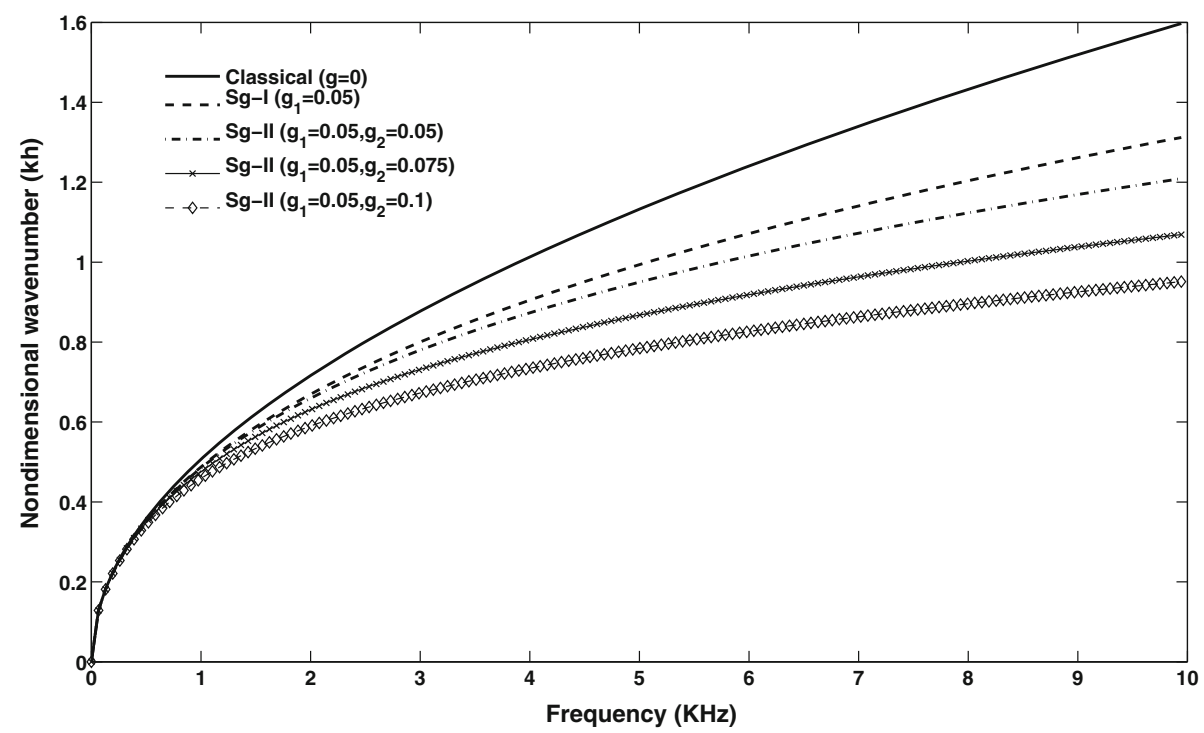

Fig. 7 Comparison of spectrum relation

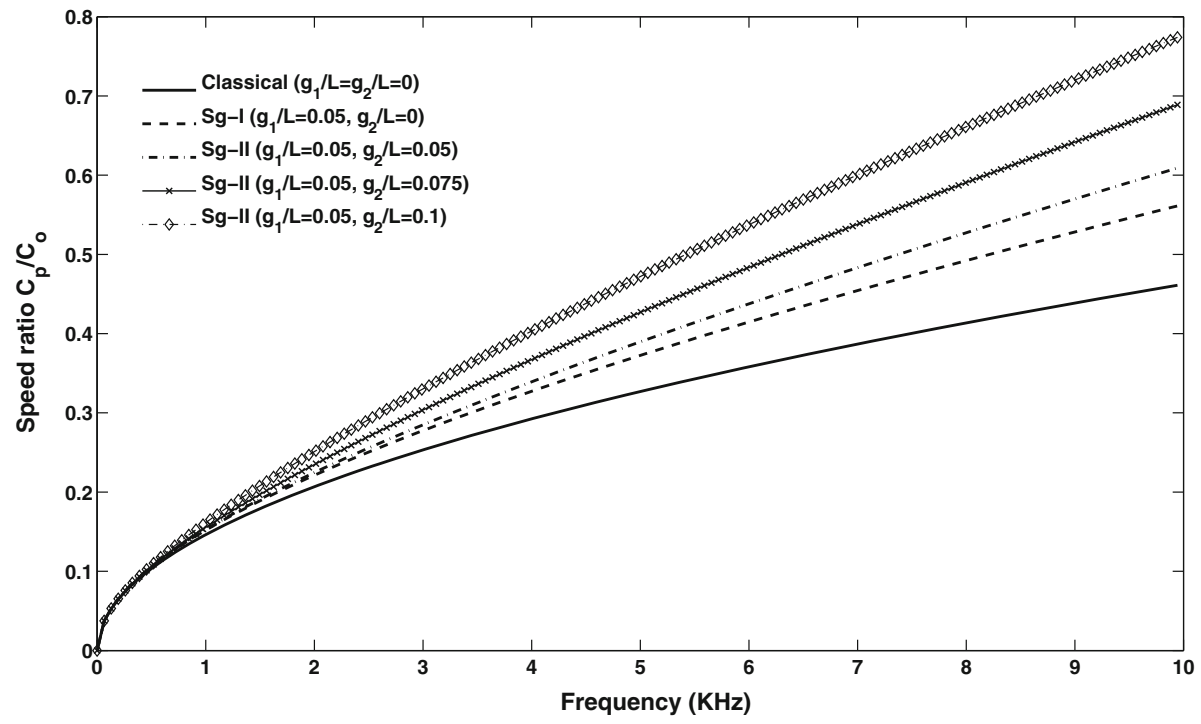

Fig. 8 Comparison of dispersion relation

presented for different boundary conditions to ascertain the gradient effect on the structural behaviour. Using the numerical Laplace transform a spectral element is formulated to study the scale effects on the dynamic response. Further, the influence of gradient effects on wave propagation characteristics is also examined based on the derived spectrum and dispersion relations. The gradient effects on the structural response are assessed and compared with the first strain gradient and classical beam theories. It is concluded that the second strain gradient theory exhibits stiffer behaviour in comparison to the first strain gradient and classical theories. The beam deflections decrease whereas frequencies and buckling load increase with higher gradient coefficients in comparison to first strain gradient theory. The amplitude of the forced response decreases and a shift is noticed to smaller time values as the gradient coefficient increases. Furthermore, the second strain gradient theory shows a dispersive behaviour, and for a given frequency, the wavenumber decreases and phase speed increases for increasing length scale parameters as compared to the first strain gradient beam theory. 


\section{Appendix}

A. Stiffness matrix and force vector for static analysis of second strain gradient Euler-Bernoulli beam

The following is the list of stiffness matrices and load vectors for different boundary conditions:

(a) Simply supported beam:

$$
[K]=\left[\begin{array}{cccccccc}
1 & 0 & 0 & 0 & 1 & 1 & 1 & 1 \\
1 & L & L^{2} & L^{3} & e^{m_{1} L} & e^{m_{2} L} & e n_{1} L & e^{n_{2} L} \\
0 & 0 & 2 & 0 & a_{11} & a_{12} & a_{13} & a_{14} \\
0 & 0 & 2 & 6 L & b_{11} & b_{12} & b_{13} & b_{14} \\
0 & 0 & 2 & 0 & m_{1}^{2} & m_{2}^{2} & n_{1}^{2} & n_{2}^{2} \\
0 & 0 & 2 & 6 L & m_{1}^{2} e^{m_{1} L} & m_{2}^{2} e^{m_{2} L} & n_{1}^{2} e^{n_{1} L} & n_{2}^{2} e^{n_{2} L} \\
0 & 0 & 0 & 6 & m_{1}^{3} & m_{2}^{3} & n_{1}^{3} & n_{2}^{3} \\
0 & 0 & 0 & 6 & m_{1}^{3} e^{m_{1} L} & m_{2}^{3} e^{m_{2} L} & n_{1}^{3} e^{n_{1} L} & n_{2}^{3} e^{n_{2} L}
\end{array}\right], \quad\{f\}=\left\{\begin{array}{c}
0 \\
-q L^{4} / 24 E I \\
g_{1}^{2} q / E I \\
g_{1}^{2} q / E I-q L^{2} / 2 E I \\
0 \\
-q L^{2} / 2 E I \\
0 \\
-q L / E I
\end{array}\right\},
$$

where

$$
\begin{aligned}
& a_{11}=m_{1}^{2}-g_{1}^{2} m_{1}^{4}+g_{2}^{4} m_{1}^{6}, \quad a_{12}=m_{2}^{2}-g_{1}^{2} m_{2}^{4}+g_{2}^{4} m_{2}^{6}, \\
& a_{13}=n_{1}^{2}-g_{1}^{2} n_{1}^{4}+g_{2}^{4} n_{1}^{6}, \quad a_{14}=n_{2}^{2}-g_{1}^{2} n_{2}^{4}+g_{2}^{4} n_{2}^{6}, \\
& b_{11}=\left(m_{1}^{2}-g_{1}^{2} m_{1}^{4}+g_{2}^{4} m_{1}^{6}\right) e^{m_{1} L}, \quad b_{12}=\left(m_{2}^{2}-g_{1}^{2} m_{2}^{4}+g_{2}^{4} m_{2}^{6}\right) e^{m_{2} L}, \\
& b_{13}=\left(n_{1}^{2}-g_{1}^{2} n_{1}^{4}+g_{2}^{4} n_{1}^{6}\right) e^{n_{1} L}, \quad b_{14}=\left(n_{2}^{2}-g_{1}^{2} n_{2}^{4}+g_{2}^{4} n_{2}^{6}\right) e^{n_{2} L} .
\end{aligned}
$$

(b) Cantilever beam:

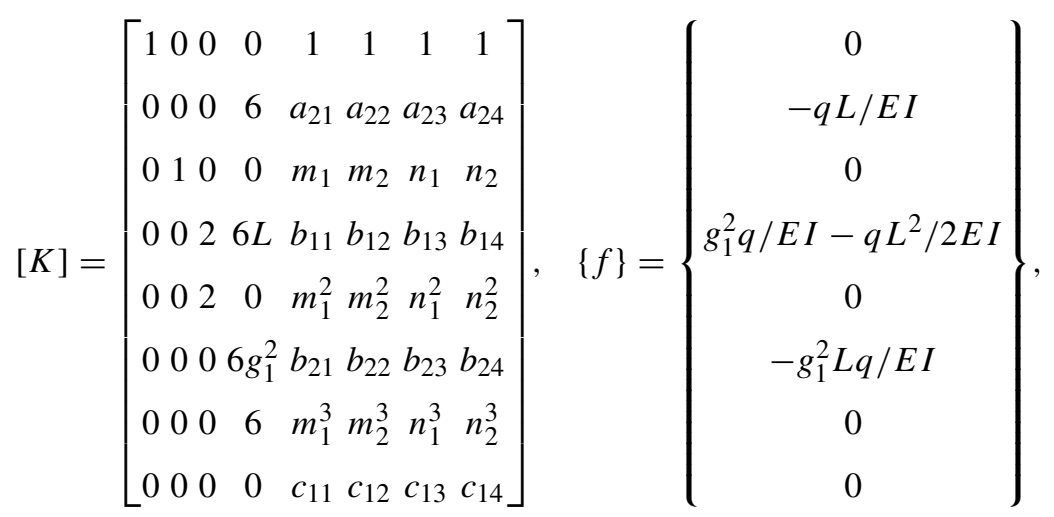

where

$$
\begin{array}{cl}
a_{21}=\left(m_{1}^{3}-g_{1}^{2} m_{1}^{5}+g_{2}^{4} m_{1}^{7}\right) e^{m_{1} L}, & a_{22}=\left(m_{2}^{3}-g_{1}^{2} m_{2}^{5}+g_{2}^{4} m_{2}^{7}\right) e^{m_{2} L}, \\
a_{23}=\left(n_{1}^{3}-g_{1}^{2} n_{1}^{5}+g_{2}^{4} n_{1}^{7}\right) e^{n_{1} L}, & a_{24}=\left(n_{2}^{3}-g_{1}^{2} n_{2}^{5}+g_{2}^{4} n_{2}^{7}\right) e^{n_{2} L}, \\
b_{21}=\left(g_{1}^{2} m_{1}^{3}-g_{2}^{4} m_{1}^{5}\right) e^{m_{1} L}, & b_{22}=\left(g_{1}^{2} m_{2}^{3}-g_{2}^{4} m_{2}^{5}\right) e^{m_{2} L}, \\
b_{23}=\left(g_{1}^{2} n_{1}^{3}-g_{2}^{4} n_{1}^{5}\right) e^{n_{1} L}, \quad b_{24}=\left(g_{1}^{2} n_{2}^{3}-g_{2}^{4} n_{2}^{5}\right) e^{n_{2} L} \\
c_{11}=g_{2}^{4} m_{1}^{4} e^{m_{1} L}, \quad c_{12}=g_{2}^{4} m_{2}^{4} e^{m_{2} L}, \quad c_{13}=g_{2}^{4} n_{1}^{4} e^{n_{1} L}, \quad c_{14}=g_{2}^{4} n_{2}^{4} e^{n_{2} L}, \\
h_{21}=\left(m_{1}^{2}-g_{1}^{2} m_{1}^{4}+g_{2}^{4} m_{1}^{6}\right) e^{m_{1} L}, \quad h_{22}=\left(m_{2}^{2}-g_{1}^{2} m_{2}^{4}+g_{2}^{4} m_{2}^{6}\right) e^{m_{2} L}, \\
h_{23}=\left(n_{1}^{2}-g_{1}^{2} n_{1}^{4}+g_{2}^{4} n_{1}^{6}\right) e^{n_{1} L}, \quad h_{24}=\left(n_{2}^{2}-g_{1}^{2} n_{2}^{4}+g_{2}^{4} n_{2}^{6}\right) e^{n_{2} L} .
\end{array}
$$


(c) Clamped beam:

$$
[K]=\left[\begin{array}{cccccccc}
1 & 0 & 0 & 0 & 1 & 1 & 1 & 1 \\
1 & L & L^{2} & L^{3} & e^{m_{1} L} & e^{m_{2} L} & e^{n_{1} L} & e^{n_{2} L} \\
0 & 1 & 0 & 0 & m_{1} & m_{2} & n_{1} & n_{2} \\
0 & 1 & 2 L & 3 L^{2} & m_{1} e^{m_{1} L} & m_{2} e^{m_{2} L} & n_{1} e^{n_{1} L} & n_{2} e^{n_{2} L} \\
0 & 0 & 2 & 0 & m_{1}^{2} & m_{2}^{2} & n_{1}^{2} & n_{2}^{2} \\
0 & 0 & 2 & 6 L & m_{1}^{2} e^{m_{1} L} & m_{2}^{2} e^{m_{2} L} & n_{1}^{2} e^{n_{1} L} & n_{2}^{2} e^{n_{2} L} \\
0 & 0 & 0 & 6 & m_{1}^{3} & m_{2}^{3} & n_{1}^{3} & n_{2}^{3} \\
0 & 0 & 0 & 6 & m_{1}^{3} e^{m_{1} L} & m_{3}^{2} e^{m_{2} L} & n_{1}^{3} e^{n_{1} L} & n_{2}^{3} e^{n_{2} L}
\end{array}\right], \quad\{f\}=\left\{\begin{array}{c}
0 \\
-q L^{4} / 24 E I \\
0 \\
-q L^{3} / 6 E I \\
0 \\
-q L^{2} / 2 E I \\
0 \\
-q L / E I
\end{array}\right\}
$$

B. Frequency equations for free vibration analysis of second strain gradient Euler-Bernoulli beam

The following are the frequency equations for different boundary conditions:

(a) Simply supported beam:

$$
[F(\omega)]=\left[\begin{array}{cccccccc}
1 & 1 & 1 & 1 & 1 & 1 & 1 & 1 \\
e^{\left(\alpha_{1} L\right)} & e^{\left(\alpha_{2} L\right)} & e^{\left(\alpha_{3} L\right)} & e^{\left(\alpha_{4} L\right)} & e^{\left(\alpha_{5} L\right)} & e^{\left(\alpha_{6} L\right)} & e^{\left(\alpha_{7} L\right)} & e^{\left(\alpha_{8} L\right)} \\
\alpha_{1}{ }^{2} & \alpha_{2}{ }^{2} & \alpha_{3}^{2} & \alpha_{4}^{2} & \alpha_{5}^{2} & \alpha_{6}^{2} & \alpha_{7}^{2} & \alpha_{8}{ }^{2} \\
t_{1} & t_{2} & t_{3} & t_{4} & t_{5} & t_{6} & t_{7} & t_{8} \\
t_{1} e^{\left(\alpha_{1} L\right)} & t_{2} e^{\left(\alpha_{2} L\right)} & t_{3} e^{\left(\alpha_{3} L\right)} & t_{4} e^{\left(\alpha_{4} L\right)} & t_{5} e^{\left(\alpha_{5} L\right)} & t_{6} e^{\left(\alpha_{6} L\right)} & t_{7} e^{\left(\alpha_{7} L\right)} & t_{8} e^{\left(\alpha_{8} L\right)} \\
\alpha_{1}^{3} & \alpha_{2}^{3} & \alpha_{3}^{3} & \alpha_{4}^{3} & \alpha_{5}^{3} & \alpha_{6}^{3} & \alpha_{7}^{3} & \alpha_{8}^{3} \\
\alpha_{1}^{3} e^{\left(\alpha_{1} L\right)} & \alpha_{2}^{3} e^{\left(\alpha_{2} L\right)} & \alpha_{3}^{3} e^{\left(\alpha_{3} L\right)} & \alpha_{4}^{3} e^{\left(\alpha_{4} L\right)} & \alpha_{5}^{3} e^{\left(\alpha_{5} L\right)} & \alpha_{6}^{3} e^{\left(\alpha_{6} L\right)} & \alpha_{7}^{3} e^{\left(\alpha_{7} L\right)} & \alpha_{8}^{3} e^{\left(\alpha_{8} L\right)}
\end{array}\right]
$$

(b) Cantilever beam:

$$
[F(\omega)]=\left[\begin{array}{cccccccc}
1 & 1 & 1 & 1 & 1 & 1 & 1 & 1 \\
\alpha_{1} & \alpha_{2} & \alpha_{3} & \alpha_{4} & \alpha_{5} & \alpha_{6} & \alpha_{7} & \alpha_{8} \\
\alpha_{1}{ }^{2} & \alpha_{2}{ }^{2} & \alpha_{3}{ }^{2} & \alpha_{4}{ }^{2} & \alpha_{5}{ }^{2} & \alpha_{6}{ }^{2} & \alpha_{7}{ }^{2} & \alpha_{8}{ }^{2} \\
\alpha_{1}{ }^{3} & \alpha_{2}{ }^{3} & \alpha_{3}{ }^{3} & \alpha_{4}{ }^{3} & \alpha_{5}{ }^{3} & \alpha_{6}{ }^{3} & \alpha_{7}{ }^{3} & \alpha_{8}{ }^{3} \\
p_{1} e^{\left(\alpha_{1} L\right)} & p_{2} e^{\left(\alpha_{2} L\right)} & p_{3} e^{\left(\alpha_{3} L\right)} & p_{4} e^{\left(\alpha_{4} L\right)} & p_{5} e^{\left(\alpha_{5} L\right)} & p_{6} e^{\left(\alpha_{6} L\right)} & p_{7} e^{\left(\alpha_{7} L\right)} & p_{8} e^{\left(\alpha_{8} L\right)} \\
r_{1} e^{\left(\alpha_{1} L\right)} & r_{2} e^{\left(\alpha_{2} L\right)} & r_{3} e^{\left(\alpha_{3} L\right)} & r_{4} e^{\left(\alpha_{4} L\right)} & r_{5} e^{\left(\alpha_{5} L\right)} & r_{6} e^{\left(\alpha_{6} L\right)} & r_{7} e^{\left(\alpha_{7} L\right)} & r_{8} e^{\left(\alpha_{8} L\right)} \\
q_{1} e^{\left(\alpha_{1} L\right)} & q_{2} e^{\left(\alpha_{2} L\right)} & q_{3} e^{\left(\alpha_{3} L\right)} & q_{4} e^{\left(\alpha_{4} L\right)} & q_{5} e^{\left(\alpha_{5} L\right)} & q_{6} e^{\left(\alpha_{6} L\right)} & q_{7} e^{\left(\alpha_{7} L\right)} & q_{8} e^{\left(\alpha_{8} L\right)} \\
\alpha_{1}^{4} e^{\left(\alpha_{1} L\right)} & \alpha_{2}^{4} e^{\left(\alpha_{2} L\right)} & \alpha_{3}^{4} e^{\left(\alpha_{3} L\right)} & \alpha_{4}^{4} e^{\left(\alpha_{4} L\right)} & \alpha_{5}^{4} e^{\left(\alpha_{5} L\right)} & \alpha_{6}^{4} e^{\left(\alpha_{6} L\right)} & \alpha_{7}^{4} e^{\left(\alpha_{7} L\right)} & \alpha_{8}^{4} e^{\left(\alpha_{8} L\right)}
\end{array}\right]
$$


(c) Clamped beam:

$$
[F(\omega)]=\left[\begin{array}{cccccccc}
1 & 1 & 1 & 1 & 1 & 1 & 1 & 1 \\
e^{\left(\alpha_{1} L\right)} & e^{\left(\alpha_{2} L\right)} & e^{\left(\alpha_{3} L\right)} & e^{\left(\alpha_{4} L\right)} & e^{\left(\alpha_{5} L\right)} & e^{\left(\alpha_{6} L\right)} & e^{\left(\alpha_{7} L\right)} & e^{\left(\alpha_{8} L\right)} \\
\alpha_{1} & \alpha_{2} & \alpha_{3} & \alpha_{4} & \alpha_{5} & \alpha_{6} & \alpha_{7} & \alpha_{8} \\
\alpha_{1} e^{\left(\alpha_{1} L\right)} & \alpha_{2} e^{\left(\alpha_{2} L\right)} & \alpha_{3} e^{\left(\alpha_{3} L\right)} & \alpha_{4} e^{\left(\alpha_{4} L\right)} & \alpha_{5} e^{\left(\alpha_{5} L\right)} & \alpha_{6} e^{\left(\alpha_{6} L\right)} & \alpha_{7} e^{\left(\alpha_{7} L\right)} & \alpha_{8} e^{\left(\alpha_{8} L\right)} \\
\alpha_{1}{ }^{2} & \alpha_{2}{ }^{2} & \alpha_{3}{ }^{2} & \alpha_{4}{ }^{2} & \alpha_{5}{ }^{2} & \alpha_{6}{ }^{2} & \alpha_{7}{ }^{2} & \alpha_{8}{ }^{2} \\
\alpha_{1}^{2} e^{\left(\alpha_{1} L\right)} & \alpha_{2}^{2} e^{\left(\alpha_{2} L\right)} & \alpha_{3}^{2} e^{\left(\alpha_{3} L\right)} & \alpha_{4}^{2} e^{\left(\alpha_{4} L\right)} & \alpha_{5}^{2} e^{\left(\alpha_{5} L\right)} & \alpha_{6}^{2} e^{\left(\alpha_{6} L\right)} & \alpha_{7}^{2} e^{\left(\alpha_{7} L\right)} & \alpha_{8}^{2} e^{\left(\alpha_{8} L\right)} \\
\alpha_{1}{ }^{3} & \alpha_{2}{ }^{3} & \alpha_{3}{ }^{3} & \alpha_{4}{ }^{3} & \alpha_{5}{ }^{3} & \alpha_{6}{ }^{3} & \alpha_{7}{ }^{3} & \alpha_{8}{ }^{3} \\
\alpha_{1}^{3} e^{\left(\alpha_{1} L\right)} & \alpha_{2}^{3} e^{\left(\alpha_{2} L\right)} & \alpha_{3}^{3} e^{\left(\alpha_{3} L\right)} & \alpha_{4}^{3} e^{\left(\alpha_{4} L\right)} & \alpha_{5}^{3} e^{\left(\alpha_{5} L\right)} & \alpha_{6}^{3} e^{\left(\alpha_{6} L\right)} & \alpha_{7}^{3} e^{\left(\alpha_{7} L\right)} & \alpha_{8}^{3} e^{\left(\alpha_{8} L\right)}
\end{array}\right],
$$

where

$$
\begin{aligned}
& t_{1}=\left(-g_{1}^{2} \alpha_{1}^{4}+g_{2}^{4} \alpha_{1}^{6}\right), \quad t_{2}=\left(-g_{1}^{2} \alpha_{2}^{4}+g_{2}^{4} \alpha_{2}^{6}\right), \quad t_{3}=\left(-g_{1}^{2} \alpha_{3}^{4}+g_{2}^{4} \alpha_{3}^{6}\right), \\
& t_{4}=\left(-g_{1}^{2} \alpha_{4}^{4}+g_{2}^{4} \alpha_{4}^{6}\right), \quad t_{5}=\left(-g_{1}^{2} \alpha_{5}^{4}+g_{2}^{4} \alpha_{5}^{6}\right), \quad t_{6}=\left(-g_{1}^{2} \alpha_{6}^{4}+g_{2}^{4} \alpha_{6}^{6}\right), \\
& t_{7}=\left(-g_{1}^{2} \alpha_{7}^{4}+g_{2}^{4} \alpha_{7}^{6}\right), \quad t_{8}=\left(-g_{1}^{2} \alpha_{8}^{4}+g_{2}^{4} \alpha_{8}^{6}\right), \\
& p_{1}=\left(\alpha_{1}^{3}-g_{1}^{2} \alpha_{1}{ }^{5}+g_{2}^{4} \alpha_{1}{ }^{7}\right), \quad p_{2}=\left(\alpha_{2}^{3}-g_{1}^{2} \alpha_{2}{ }^{5}+g_{2}^{4} \alpha_{2}{ }^{7}\right), \\
& p_{3}=\left(\alpha_{3}^{3}-g_{1}^{2} \alpha_{3}{ }^{5}+g_{2}^{4} \alpha_{3}{ }^{7}\right), \quad p_{4}=\left(\alpha_{4}^{3}-g_{1}^{2} \alpha_{4}{ }^{5}+g_{2}^{4} \alpha_{4}{ }^{7}\right), \\
& p_{5}=\left(\alpha_{5}^{3}-g_{1}^{2} \alpha_{5}^{5}+g_{2}^{4} \alpha_{5}{ }^{7}\right), \quad p_{6}=\left(\alpha_{6}^{3}-g_{1}^{2} \alpha_{6}{ }^{5}+g_{2}^{4} \alpha_{6}{ }^{7}\right), \\
& p_{7}=\left(\alpha_{7}^{3}-g_{1}^{2} \alpha_{7}^{5}+g_{2}^{4} \alpha_{7}^{7}\right), \quad p_{8}=\left(\alpha_{8}^{3}-g_{1}^{2} \alpha_{8}^{5}+g_{2}^{4} \alpha_{8}{ }^{7}\right) \text {. } \\
& r_{1}=\left(\alpha_{1}^{2}-g_{1}^{2} \alpha_{1}{ }^{4}+g_{2}^{4} \alpha_{1}^{6}\right), \quad r_{2}=\left(\alpha_{2}^{2}-g_{1}^{2} \alpha_{2}^{4}+g_{2}^{4} \alpha_{2}{ }^{6}\right) \text {, } \\
& r_{3}=\left(\alpha_{3}^{2}-g_{1}^{2} \alpha_{3}{ }^{4}+g_{2}^{4} \alpha_{3}{ }^{6}\right), \quad r_{4}=\left(\alpha_{4}^{2}-g_{1}^{2} \alpha_{4}{ }^{4}+g_{2}^{4} \alpha_{4}{ }^{6}\right) \text {, } \\
& r_{5}=\left(\alpha_{5}^{2}-g_{1}^{2} \alpha_{5}{ }^{4}+g_{2}^{4} \alpha_{5}{ }^{6}\right), \quad r_{6}=\left(k_{6}^{2}-g_{1}^{2} \alpha_{6}{ }^{4}+g_{2}^{4} \alpha_{6}{ }^{6}\right), \\
& r_{7}=\left(\alpha_{7}^{2}-g_{1}^{2} \alpha_{7}^{4}+g_{2}^{4} \alpha_{7}{ }^{6}\right), \quad r_{8}=\left(\alpha_{8}^{2}-g_{1}^{2} \alpha_{8}^{4}+g_{2}^{4} \alpha_{8}^{6}\right) \text {, } \\
& q_{1}=\left(g_{1}^{2} \alpha_{1}{ }^{3}-g_{2}^{4} \alpha_{1}{ }^{5}\right), \quad q_{2}=\left(g_{1}^{2} \alpha_{2}{ }^{3}-g_{2}^{4} \alpha_{2}{ }^{5}\right), \\
& q_{3}=\left(g_{1}^{2} \alpha_{3}{ }^{3}-g_{2}^{4} \alpha_{3}{ }^{5}\right), \quad q_{4}=\left(g_{1}^{2} \alpha_{4}{ }^{3}-g_{2}^{4} \alpha_{4}{ }^{5}\right), \\
& q_{5}=\left(g_{1}^{2} \alpha_{5}{ }^{3}-g_{2}^{4} \alpha_{5}{ }^{5}\right), \quad q_{6}=\left(g_{1}^{2} \alpha_{6}{ }^{3}-g_{2}^{4} \alpha_{6}{ }^{5}\right), \\
& q_{7}=\left(g_{1}^{2} \alpha_{7}^{3}-g_{2}^{4} \alpha_{7}^{5}\right), \quad q_{8}=\left(g_{1}^{2} \alpha_{8}{ }^{3}-g_{2}^{4} \alpha_{8}{ }^{5}\right),
\end{aligned}
$$

C. Geometric stiffness matrix for buckling analysis of second strain gradient Euler-Bernoulli beam

The following are the geometric stiffness matrices for different boundary conditions:

(a) Simply supported beam:

$$
[G(P)]=\left[\begin{array}{cccccccc}
1 & 0 & 1 & 1 & 1 & 1 & 1 & 1 \\
1 & L & e^{\left(m_{1} L\right)} & e^{\left(m_{2} L\right)} & e^{\left(m_{3} L\right)} & e^{\left(n_{1} L\right)} & e^{\left(n_{2} L\right)} & e^{\left(n_{3} L\right)} \\
0 & 0 & m_{1}^{2} & m_{2}^{2} & m_{3}^{2} & n_{1}^{2} & n_{2}^{2} & n_{3}^{2} \\
0 & 0 & t_{3} & t_{4} & t_{5} & t_{6} & t_{7} & t_{8} \\
0 & 0 & t_{3} e^{\left(m_{1} L\right)} & t_{4} e^{\left(m_{2} L\right)} & t_{5} e^{\left(m_{3} L\right)} & t_{6} e^{\left(n_{1} L\right)} & t_{7} e^{\left(n_{2} L\right)} & t_{8} e^{\left(n_{3} L\right)} \\
0 & 0 & m_{1}^{3} & m_{2}^{3} & m_{3}^{3} & n_{1}^{3} & n_{2}^{3} & n_{3}^{3} \\
0 & 0 & m_{1}^{3} e^{\left(m_{1} L\right)} & m_{2}^{3} e^{\left(m_{2} L\right)} & n_{1}^{3} e^{\left(n_{1} L\right)} & n_{2}^{3} e^{\left(n_{2} L\right)} & n_{3}^{3} e^{\left(n_{3} L\right)} & n_{4}^{3} e^{\left(n_{4} L\right)}
\end{array}\right]
$$


(b) Cantilever beam:

$$
[G(P)]=\left[\begin{array}{cccccccc}
1 & 0 & 1 & 1 & 1 & 1 & 1 & 1 \\
0 & 1 & m_{1} & m_{2} & m_{3} & n_{1} & n_{2} & n_{3} \\
0 & 0 & m_{1}{ }^{2} & m_{2}{ }^{2} & m_{3}^{2} & n_{1}{ }^{2} & n_{2}{ }^{2} & n_{3}{ }^{2} \\
0 & 0 & m_{1}{ }^{3} & m_{2}{ }^{3} & m_{3}^{3} & n_{1}{ }^{3} & n_{2}{ }^{3} & n_{3}{ }^{3} \\
0 & 0 & p_{3} e^{\left(m_{1} L\right)} & p_{4} e^{\left(m_{2} L\right)} & p_{5} e^{\left(m_{3} L\right)} & p_{6} e^{\left(n_{1} L\right)} & p_{7} e^{\left(n_{2} L\right)} & p_{8} e^{\left(n_{3} L\right)} \\
0 & 0 & r_{3} e^{\left(m_{1} L\right)} & r_{4} e^{\left(m_{2} L\right)} & r_{5} e^{\left(m_{3} L\right)} & r_{6} e^{\left(n_{1} L\right)} & r_{7} e^{\left(n_{2} L\right)} & r_{8} e^{\left(n_{3} L\right)} \\
0 & 0 & q_{3} e^{\left(m_{1} L\right)} & q_{4} e^{\left(m_{2} L\right)} & q_{5} e^{\left(m_{3} L\right)} & q_{6} e^{\left(n_{1} L\right)} & q_{7} e^{\left(n_{2} L\right)} & q_{8} e^{\left(n_{3} L\right)} \\
0 & 0 & m_{1}^{4} e^{\left(m_{1} L\right)} & m_{2}^{4} e^{(2 L)} & m_{3}^{4} e^{\left(m_{3} L\right)} & n_{1}^{4} e^{\left(n_{1} L\right)} & n_{2}^{4} e^{\left(n_{2} L\right)} & n_{3}^{4} e^{\left(n_{3} L\right)}
\end{array}\right]
$$

(c) Clamped beam:

$$
[G(P)]=\left[\begin{array}{cccccccc}
1 & 0 & 1 & 1 & 1 & 1 & 1 & 1 \\
1 & L & e^{\left(m_{1} L\right)} & e^{\left(m_{2} L\right)} & e^{\left(m_{3} L\right)} & e^{\left(n_{1} L\right)} & e^{\left(n_{2} L\right)} & e^{\left(n_{3} L\right)} \\
0 & 1 & m_{1} & m_{2} & m_{3} & n_{1} & n_{2} & n_{3} \\
0 & 1 & m_{1} e^{\left(m_{1} L\right)} & m_{2} e^{\left(m_{2} L\right)} & m_{3} e^{\left(m_{3} L\right)} & n_{1} e^{\left(n_{1} L\right)} & n_{2} e^{\left(n_{2} L\right)} & n_{3} e^{\left(n_{3} L\right)} \\
0 & 0 & m_{1}{ }^{2} & m_{2}{ }^{2} & m_{3}{ }^{2} & n_{1}{ }^{2} & n_{2}{ }^{2} & n_{3}{ }^{2} \\
0 & 0 & m_{1}^{2} e^{\left(m_{1} L\right)} & m_{2}^{2} e^{\left(m_{2} L\right)} & m_{3}^{2} e^{\left(m_{3} L\right)} & n_{1}^{2} e^{\left(n_{1} L\right)} & n_{2}^{2} e^{\left(n_{2} L\right)} & n_{3}^{2} e^{\left(n_{3} L\right)} \\
0 & 0 & m_{1}{ }^{3} & m_{2}{ }^{3} & m_{3}^{3} & n_{1}{ }^{3} & n_{2}{ }^{3} & n_{3}{ }^{3} \\
0 & 0 & m_{1}^{3} e^{\left(m_{1} L\right)} & m_{2}^{3} e^{\left(m_{2} L\right)} & m_{3}^{3} e^{\left(m_{3} L\right)} & n_{1}^{3} e^{\left(n_{1} L\right)} & n_{2}^{3} e^{\left(n_{2} L\right)} & n_{3}^{3} e^{\left(n_{3} L\right)}
\end{array}\right]
$$

\section{References}

1. Najar, F., Choura, S., El-Borgi, S., Abdel-Rahman, E.M., Nayfeh, A.H.: Modeling and design of variable-geometry electrostatic microactuators. J. Micromech. Microeng. 15, 419-429 (2005)

2. Li, X., Bhushan, B., Takashima, K., Baek, C.W., Kim, Y.K.: Mechanical characterization of micro/nanoscale structures for MEMS/NEMS applications using nanoindentation techniques. Ultramicroscopy 97, 481-494 (2003)

3. Lin, C.H., Ni, H., Wang, X., Chang, M., Chao, Y.J., Deka, J.R., Li, X.: In situ nanomechanical characterization of singlecrystalline boron nanowires by buckling. Small 6(8), 927-931 (2010)

4. Zhu, Y., Qin, Q., Xu, F., Fan, F., Ding, Y., Zhang, T., Wang, Z.L.: Size effects on elasticity, yielding, and fracture of silver nanowires: In situ experiments. Phys. Rev. B 85(4), 045443 (2012)

5. Jiang, W., Batra, R.: Molecular statics simulations of buckling and yielding of gold nanowires deformed in axial compression. Acta Materialia 57(16), 4921-4932 (2009)

6. Wang, Z., Zu, X., Gao, F., Weber, W.J.: Atomistic simulations of the mechanical properties of silicon carbide nanowires. Phys. Rev. B 77(22), 224113 (2008)

7. Rabkin, E., Nam, H.S., Srolovitz, D.: Atomistic simulation of the deformation of gold nanopillars. Acta Materialia 55(6), 2085-2099 (2007)

8. Cosserat, E., Cosserat, F.: Theorie des corps deformables, Hermann Archives (reprint 2009)

9. Eringen, A.C., Suhubi, E.S.: Nonlinear theory of simple microelastic solids, I and II, nonlinear theory of simple microelastic solids, I and II. Int. J. Eng. Sci. 2(189-203), 389-404 (1964)

10. Polizzotto, C.: Nonlocal elasticity and related variational principles. Int. J. Solids Struct. 38, 7359-7380 (2001)

11. Eringen, A.C.: Nonlocal polar elastic continua. Int. J. Eng. Sci. 10, 1-16 (1972)

12. Eringen, A.C.: On differential equations of nonlocal elasticity and solutions of screw dislocation and surface waves. J. Appl. Phys. 54, 4703-4710 (1983)

13. Fleck, N.A., Hutchinson, J.W.: A phenomenological theory for strain gradient effects in plasticity. J. Mech. Phys. Solids 41(12), 1825-1857 (1993)

14. Fleck, N.A., Hutchinson, J.W.: A reformulation of strain gradient plasticity. J. Mech. Phys. Solids 49, $2245-2271$ (2001)

15. Mindlin, R.D.: Micro-structure in linear elasticity. Arch. Ration. Mech. Anal. 16, 52-78 (1965)

16. Mindlin, R.D., Eshel, N.: On first strain-gradient theories in linear elasticity. Int. J. Solids Struct. 4, 109-124 (1968)

17. Toupin, R.: Elastic materials with couple-stresses. Arch. Ration. Mech Anal. 11, 385-414 (1962)

18. Koiter, W.T.: Couple-stresses in the theory of elasticity, I \& II. Proc. K. Ned. Akad. Wet. (B) 67, 17-44 (1964) 
19. Askes, H., Aifantis, E.C.: Gradient elasticity in statics and dynamics: an overview of formulations, length scale identification procedures, finite element implementations and new results. Int. J. Solids Struct. 48, 1962-1990 (2011)

20. Vardoulakis, I., Sulem, J.: Bifurcation Analysis in Geomechanics. Blackie/Chapman and Hall, London (1995)

21. Askes, H., Suiker, A.S.J., Sluys, L.J.: A classification of higher-order strain-gradient models linear analysis. Arch. Appl. Mech. 72, 171-188 (2002)

22. Yang, F., Chong, A.C.M., Lam, D.C.C., Tong, P.: Experiments and theory in strain gradient elasticity. J. Mech. Phys. Solids 51, 1477-1508 (2003)

23. Lam, D.C.C., Yang, F., Chong, A.C.M., Wang, J., Tong, P.: Couple stress based strain gradient theory for elasticity. Int. J. Solids Struct. 39, 2731-2743 (2002)

24. Thai, H., Vo, T.P., Nguyen, T., Kim, S.: A review of continuum mechanics models for size-dependent analysis of beams and plates. Comput. Struct. 177(1), 196-219 (2017)

25. Ma, H.M., Gao, X.L., Reddy, J.N.: A microstructure-dependent Timoshenko beam model based on a modified couple stress theory. J. Mech. Phys. Solids. 56, 3379-3391 (2008)

26. Akgoz, B., Civalek, O.: Analysis of micro-sized beams for various boundary conditions based on the strain gradient elasticity theory. Arch. Appl. Mech. 82(3), 423-43 (2012)

27. Kong, S., Zhou, S., Nie, Z., Wang, K.: Static and dynamic analysis of micro beams based on strain gradient elasticity theory. Int. J. Eng. Sci. 47, 487-498 (2009)

28. Reddy, J.N.: Nonlocal theories for bending, buckling, and vibration of beams. Int. J. Eng. Sci. 45, 288-307 (2007)

29. Exadaktylos, G.E., Vardoulakis, I.: Microstructure in linear elasticity and scale effects: a reconsideration of basic rock mechanics and rock fracture mechanics. Tectonophysics 335, 81-109 (2001)

30. Vardoulakis, I., Exadactylos, G., Kourkoulis, S.K.: Bending of marble with intrinsic length scales: a gradient theory with surface energy and size effects. J. Phys. IV. 8, 399-406 (1998)

31. Ru, C.Q., Aifantis, E.C.: A simple approach to solve boundary value problems in gradient elasticity. Acta Mech. 101, 59-68 (1993)

32. Tsepoura, K.G., Papargyri-Beskou, S., Polyzos, D., Beskos, D.E.: Static and dynamic analysis of gradient elastic bars in tension. Arch. Appl. Mech. 72, 483-497 (2002)

33. Georgiadis, H.G., Anagnostou, D.S.: Problems of Flamant-Boussinesq and Kelvin type in dipolar gradient elasticity. J. Elast. 90, 71-98 (2008)

34. Gao, X.L., Ma, H.M.: Greens function and Eshelbys tensor based on a simplified strain gradient elasticity theory. Acta Mech. 207, 163-181 (2009)

35. Georgiadis, H.G., Vardoulakis, I., Lykotrafitis, G.: Torsional surface waves in a gradient-elastic half-space. Wave Motion 31, $333-348(2000)$

36. Georgiadis, H.G., Vardoulakis, I., Velgaki, E.G.: Dispersive Rayleigh-wave propagation in microstructured solids characterized by dipolar gradient elasticity. J. Elast. 74, 17-45 (2004)

37. Polyzos, D., Fotiadis, D.I.: Derivation of Mindlins gradient elastic theory via simple lattice and continuum models. Int. J. Solids Struct. 49, 470-480 (2012)

38. Papargyri-Beskou, S., Tsepoura, K.G., Polyzos, D., Beskos, D.E.: Bending and stability analysis of gradient elastic beams. Int. J. Solids Struct. 40, 385-400 (2003)

39. Papargyri-Beskou, S., Polyzos, D., Beskos, D.E.: Dynamic analysis of gradient elastic flexural beams. Struct. Eng. Mech. 15(6), 705-716 (2003)

40. Lazopoulos, A.K.: Dynamic response of thin strain gradient elastic beams. Int. J. Mech. Sci. 58, 27-33 (2012)

41. Lazopoulos, K.A., Lazopoulos, A.K.: Bending and buckling of thin strain gradient elastic beams. Eur. J. Mech. A/Solids 29, 837-843 (2010)

42. Pegios, I.P., Papargyri-Beskou, S., Beskos, D.E.: Finite element static and stability analysis of gradient elastic beam structures. Acta Mech. 226, 745-768 (2015)

43. Pegios, I.P., Hatzigeorgiou, G.D.: Finite element free and forced vibration analysis of gradient elastic beam structures. Acta Mech. 229, 4817-4830 (2018)

44. Tsinopoulos, S.V., Polyzos, D., Beskos, D.E.: Static and dynamic BEM analysis of strain gradient elastic solids and structures. Comput. Mod. Eng. Sci. 86, 113-144 (2012)

45. Mindlin, R.D.: Second gradient of strain and surface-tension in linear elasticity. Int. J. Solids Struct. 1, 417-438 (1965)

46. Chien, HWu: Cohesive elasticity and surface phenomena. Q. Appl. Math. 1, 73-103 (1992)

47. Amiot, F.: An EulerBernoulli second strain gradient beam theory for cantilever sensors. Philos. Mag. Lett. 93(4), 204-212 (2013)

48. Shodja, H.M., Ahmadpoor, F., Tehranchi, A.: Calculation of the additional constants for FCC materials in second strain gradient elasticity: behavior of a nano-size Bernoulli-Euler beam with surface effects. J. Appl. Mech. 79, 021008 (2012)

49. Ojaghnezhad, F., Shodja, H.M.: A combined first principles and analytical determination of the modulus of cohesion, surface energy and the additional constants in the second strain gradient elasticity. Int. J. Solids Struct. 50, 3967-3974 (2013)

50. Cordero, N.M., Forest, S., Busso, E.P.: Second strain gradient elasticity of nano-objects. J. Mech. Phys. Solids 97, 92-124 (2016)

51. Polyzos, D., Fotiadis, D.I.: Derivation of Mindlins first and second strain gradient elastic theory via simple lattice and continuum models. Int. J. Solids Struct. 49, 470-480 (2012)

52. Polizzotto, C.: A gradient elasticity theory for second-grade materials and higher order inertia. Int. J. Solids Struct. 49, 2121-2137 (2012)

53. Polizzotto, C.: A second strain gradient elasticity theory with second velocity gradient inertia Part I: constitutive equations and quasi-static behavior. Int. J. Solids Struct. 50, 3749-3765 (2013)

54. Polizzotto, C.: A second strain gradient elasticity theory with second velocity gradient inertia Part II: dynamic behaviour. Int. J. Solids Struct. 50, 3766-3777 (2013)

55. Forest, S., Cordero, N.M., Busso, E.P.: First vs. second gradient of strain theory for capillarity effects in an elastic fluid at small length scales. Comput. Mater. Sci. 50, 1299-1304 (2011) 
56. Momeni, S.A., Asghari, M.: The second strain gradient functionally graded beam formulation. Comput. Struct. 188, 15-24 (2018)

57. Lazar, M., Maugin, G.A., Aifantis, E.C.: Dislocations in second strain gradient elasticity. Int. J. Solids Struct. 43, 1787-1817 (2006)

58. Polizzotto, C.: Gradient elasticity and non standard boundary conditions. Int. J. Solids Struct. 40, 7399-7423 (2003)

59. Deng, S., Liu, J., Liang, N.: Wedge and twist disclinations in second strain gradient elasticity. Int. J. Solids Struct. 44, 3646-3665 (2007)

60. Polizzotto, C.: Surface effects, boundary conditions and evolution laws within second strain gradient plasticity. Int. J. Plast. 60, 197-216 (2014)

61. Zhang, X., Jiao, K., Sharma, P., Yakobson, B.I.: An atomistic and non-classical continuum field theoretic perspective of elastic interactions between defects (force dipole) of various symmetries and application to graphene. J. Mech. Phys. Solids. 54, 2304-2329 (2006)

62. Lazar, M.: The fundamentals of nano-singular dislocations in the theory of gradient elasticity: dislocation loops and straight dislocations. Int. J. Solids Struct. 50, 352-362 (2013)

63. Ishaquddin, Md., Gopalakrishnan, S.: Differential quadrature element for second strain gradient beam theory. arXiv: 1807.0 $8622(2016)$

64. Ishaquddin, Md., Gopalakrishnan, S.: Novel weak form quadrature elements for second strain gradient Euler-Bernoulli beam theory. arXiv:1807.08625 (2016)

65. Ramirez, A., Gomez, P., Moreno, P., Gutierrez, A.: Frequency domain analysis of electromagnetic transients through the numerical Laplace transforms. In: Proceedings of IEEE Power Engineering Society of America, Denver, CO, USA, vol. 1, pp. 1136-1139 (2004)

66. Moreno, P., Ramirez, A.: Implementation of numerical Laplace transform: a review. IEEE Trans. Power Deliv. 23(4), 2599-2609 (2008)

67. Igawa, H., Komatsu, K., Yamaguchi, I., Kasai, T.: Wave propagation analysis of frame structures using the spectral element method. J. Sound Vib. 277, 1071-1081 (2003)

68. Blais, J.F., Cimmino, M., Ross, A., Granger, D.: Suppression of time aliasing in the solution of the equations of motion of an impacted beam with partial constrained layer damping. J. Sound Vib. 326, 870-882 (2009)

69. Kishor, D.K., Gopalakrishnan, S., Ganguli, R.: Three-dimensional sloshing: A consistent finite element approach. Int. J. Numer. Methods Fluids. 66(3), 345-376 (2011)

70. Doyle, J.F., Farris, T.N.: A spectrally formulated finite element for flexural wave propagation in beams. Int. J. Anal. Exp. Modal Anal. 5, 13-23 (1990)

71. Doyle, J.F.: Wave Propagation in Structures, 2nd edn. Springer, New York (1997)

72. Gopalakrishnan, S., Chakraborty, A., Mahapatra, D.R.: Spectral Finite Element Method. Springer, London (2008)

73. Wilcox, D.J.: Numerical Laplace transformation and inversion. Int. J. Electr. Eng. Educ. 15(3), 247-265 (1978)

74. Wedepohl, L.M.: Power systems transients: errors incurred in the numerical inversion of the Laplace transform. In: Proceedings of Midwest Symposium on Circuits and Systems, Puebla, Mex, pp. 174-178 (1983)

75. Murthy, M.V.V.S., Gopalakrishnan, S., Nair, P.S.: Signal wrap-around free spectral element formulation for multiply connected finite 1D waveguides. J. Aerosp. Sci. Technol. 63(1), 72-88 (2011)

76. Patra, A.K., Gopalakrishnan, S., Ganguli, R.: A spectral multiscale method for wave propagation analysis: atomistic continuum coupled simulation. Comput. Methods Appl. Mech. Eng. 278, 744-764 (2014)

77. Gopalakrishnan, S.: Wave Propagation in Materials and Structures. Taylor \& Francis Group/CRC Press, New York (2017)

78. Cooley, J.W., Tukey, O.W.: An algorithm for the machine clculation of complex Fourier series. Math. Comput. 19(90), 297-301 (1965)

79. Cooley, J.W., Lewis, P.A.W., Welch, P.D.: The fast Fourier transform algorithm: programming considerations in the calculation of sine, cosine and laplace transforms. J. Sound Vib. 12, 315-337 (1970)

80. Kitahara, M.: Boundary Integral Equation Methods in Eigenvalue Problems of Elastodynamics and Thin Plates. Elsevier, Amsterdam (1985)

81. Reddy, J.N.: Energy Principles and Variational Methods in Applied Mechanics, 2nd edn. Wiley, New York (2002)

Publisher's Note Springer Nature remains neutral with regard to jurisdictional claims in published maps and institutional affiliations. 